\title{
HIF2a Exerts Carcinogenesis in Clear Cell Renal Cell Carcinoma by Targeting High Expression of NUDT1 to Inhibit Oxidative Stress
}

Jian Shi

Wuhan Union Hospital

Zhiyong Xiong

Wuhan Union Hospital

Keshan Wang

Wuhan Union Hospital

Changfei Yuan

Wuhan Union Hospital

Yu Huang

Wuhan Union Hospital

Wen Xiao

Wuhan Union Hospital

Huageng Liang

Wuhan Union Hospital

Tianbo Xu

Wuhan Union Hospital

Hongmei Yang

Huazhong University of Science and Technology

Ke Chen

Wuhan Union Hospital

Xiao-ping Zhang ( $\nabla$ xzhang@hust.edu.cn )

Departmento of Urology, Union Hospital, Tongji Medical College, Huazhong Universtiy of Science and Technology

\section{Research}

Keywords: Clear cell renal cell carcinoma, oxidative stress, reactive oxygen species, nucleoside diphosphate linked moiety X-type motif 1 (NUDT1), HIF2a

Posted Date: September 23rd, 2020

DOl: https://doi.org/10.21203/rs.3.rs-77369/v1 
License: (c) (i) This work is licensed under a Creative Commons Attribution 4.0 International License. Read Full License 


\section{Abstract}

Background: Emerging evidence highlights the important roles of HIF2a in the development and progression of clear cell renal cell carcinoma (ccRCC). Recently, oxidative stress has been shown to play a vital role in an increasing number of tumor types. However, the relationship between the two factors in ccRCC is still unclear. The aim of this work is to study the role of oxidative stress in ccRCC and its relationship with HIF2a.

Methods: Molecular screening and bioinformatics analysis in cCRCC based on data from the TCGA database. Regulated pathways were investigated by qRT-PCR, immunoblot analysis, luciferase reporter and chromatin immunoprecipitation (ChIP) assays. A series of functional analyses were conducted in cell lines and xenograft models.

Results: By screening three independent oxidative stress-related gene sets and the whole transcriptome sequencing data obtained after HIF2a knockdown, NUDT1 was discovered as a bridge molecule mediating the interrelationship between HIF2 $a$ and oxidative stress. Bioinformatics and experimental studies have found that NUDT1 is upregulated in CCRCC and has significant prognostic implications. Mechanistically, HIF2a directly increases NUDT1 expression by binding to HIF2a response elements in the NUDT1 promoter region. Reducing the expression of NUDT1 can significantly increase the level of oxidative stress in ccRCC cells, resulting in the inhibition of the carcinogenic effect of HIF2a.

Conclusions: Our research systematically identify the regulatory mechanisms of HIF2a and oxidative stress in ccRCC for the first time. It provides a new understanding of cCRCC and can help us creating new strategies for its treatment.

\section{Introduction}

Renal cell carcinoma (RCC) currently accounts for approximately $3 \%$ of the world's cancer diagnoses (1). Clear cell renal cell carcinoma (ccRCC) is the most common type of renal cell carcinoma, accounting for approximately $85 \%$. In $90 \%$ of ccRCC, the pathogenesis is characterized by constitutive activation of hypoxia-inducible factor (HIF) due to the lack of von Hippel-Lindau (VHL) tumor suppressor, which is required for oxygen $\left(\mathrm{O}_{2}\right)$-dependent inhibition of hypoxia-inducible factors (HIF) signaling (2). The process of HIF regulation is coordinated by two HIFa subunits (HIF1a and HIF2a), which form a complex to direct the transcriptional activation of hundreds of genes promoting adaptation to hypoxia that are associated with tumor development (3). Current research shows that HIF1a plays a role as a tumor suppressor gene, while HIF2a is one of the most important oncogenes, directly promoting the progression of ccRCC by activating its target genes (4-6).

Oxidative stress is defined as a kind of cellular state in which the reactive oxygen species (ROS) level is increased and eclipses the antioxidant defense mechanisms in cells. Oxidative stress is characterized by an imbalance in the production of cellular oxidants and the removal of their byproducts $(7,8)$. Regulation of intracellular ROS levels is essential for cell homeostasis, as different ROS levels can induce different 
biological responses. At low and medium levels, ROS can serve as a signaling molecule to maintain cell proliferation and differentiation. At high levels, ROS can affect the structure and function of critical cellular molecules such as proteins, lipids, and DNA, which influence the growth, mutation of the cells and chromosome instability. These changes can ultimately yield neoplasm formation (9-11). Previous studies had confirmed that the HIF family is inextricably linked to oxidative stress. The expression of HIF1a in cells is associated with ROS-induced carcinogenesis in several human tumors (12). In addition, HIF2 $a$ is potentially linked to oxidative stress, which can regulate erythropoietin and the mitochondrial matrix protein superoxide dismutase 2 (SOD2)(13-15). However, the specific mechanism and role between HIF2 $a$ and oxidative stress are still unclear.

Nucleoside diphosphate linked moiety X-type motif 1 (NUDT1) is an 18kD nudix pyrophosphatase with substrate specificity for 8-oxo-dGTP and 8-oxo-GTP $(16,17)$. NUDT1-dependent increases have been observed in tumors with elevated oxidation levels and a tendency for DNA damage $(18,19)$. NUDT1 is essential for RAS/ROS-related transformation and proliferation in tumorigenic cells (20). It can reduce the effect of oxidative stress on cells (especially tumor cells), and it also has a DNA maintenance function, which protects tumor cells from genomic DNA breaks and related negative impacts on viability (21-23). Furthermore, numerous studies have shown that high expression of NUDT1 in human lung tumors (24), colorectal tumors (25), esophageal squamous cell carcinomas (26) and glioblastomas (27) is associated with greater malignancy and poor prognosis. However, it has never been studied in ccRCC.

In this study, we identified the effects of oxidative stress on the progression of ccRCC and analyzed the role and association between HIF2 $a$ and NUDT1 during the progression of ccRCC. It provides new strategies for the treatment of ccRCC.

\section{Materials And Methods}

\section{Cell lines and cell culture}

HK-2, 786-0, A-498, ACHN and Caki-1 cell lines were purchased from the American Type Culture Collection (ATCC, USA). All cells were maintained in Dulbecco's modified Eagle's medium (HyClone; GE Healthcare, Logan, UT, USA) supplemented with 10\% fetal bovine serum (FBS; Gibco; Thermo Fisher Scientific, Inc., Waltham, MA, USA) and $1 \%$ penicillin-streptomycin solution. Cells were maintained at $37^{\circ} \mathrm{C}$ in an incubator containing $5 \% \mathrm{CO} 2$.

\section{Human ccRCC tissue samples}

All samples were obtained from patients with ccRCC who underwent partial or radical nephrectomy from 2016 to 2018 at Union Hospital of Huazhong University of Science and Technology (Wuhan, China). The excised tissues were immediately frozen in liquid nitrogen for subsequent experiments. No patients who underwent surgery received any antitumor treatment before surgery. The samples were obtained with written informed consent from each patient. This study was approved by the Huazhong University of Science and Technology Committee. 


\section{Immunohistochemistry and immunofluorescence staining}

Four-micrometer sections prepared from paraffin-embedded C4-2 tissues were used for immunohistochemical staining. The sections were sequentially deparaffinized, rehydrated, and incubated for antigen retrieval. Blocking was performed with fetal bovine serum after the sections were incubated with $3 \% \mathrm{H}_{2} \mathrm{O}_{2}$ at room temperature for $15 \mathrm{~min}$. Then, the sections were incubated with primary antibodies overnight at $4^{\circ} \mathrm{C}$. Immunodetection was performed with an HRP-conjugated secondary antibody for $1 \mathrm{~h}$. DAB was used to visualize the immune complexes. Finally, nuclei were counterstained with hematoxylin.

For immunofluorescent staining, cells were fixed in $4 \%$ paraformaldehyde, permeated with $0.5 \%$ Triton X100 , and blocked with $5 \%$ goat serum in PBS for 1 hour at $37^{\circ} \mathrm{C}$, which was followed by incubation with primary antibodies. Finally, Alexa Fluor 488-conjugated Donkey Anti-Rabbit lgG ( $\mathrm{H}+\mathrm{L})$ (Abclonal, AS035) was used as the secondary antibody to generate a visible signal. DAPI was used to stain nuclei.

\section{RNA Extraction, cDNA Synthesis and qPCR}

Total RNA was extracted from tissues with TRIzol reagent (Thermo, Massachusetts, USA) according to the instruction manual, and $1 \mu \mathrm{g}$ of enriched tissue or cell RNA was used in reverse transcription. A NanoDrop 2000 spectrophotometer (NanoDrop Technologies, Wilmington, USA) was used to measure the purity and concentration of the RNA solution. The housekeeping gene glyceraldehyde-3-phosphate dehydrogenase (GAPDH) was used as an internal control to standardize the variability in expression levels. SYBR Green mix (Thermo, Massachusetts, USA) was used for qPCR analysis. The specific gene primer sequences are as follows:

GAPDH: forward, 5'-CCAGAACATCATCCCTGCCT-3',

reverse, 5'-CCTGCTTCACCACCTTCTTG-3';

NUDT1: forward, 5'-GGCCAGATCGTGTTTGAGTT-3',

reverse, 5'-TGAAGCAGGAGTGGAAACCA-3';

and HIF2a: forward 5'-TCGGAGAGGAGGAAGGAGAA-3',

reverse, 5'-GAGGAGAGGAGCTTGTGTGT-3'.

\section{Transfection assay}

The following HIF2a-targeted short hairpin RNAs and NUDT1- targeted short hairpin RNAs were designed and synthesized by Genechem Co. Ltd (Shanghai, China). The constructed lentivirus was used to infect A498 or 786-0 cells according to the manufacturer's protocol. The plasmids for overexpression of NUDT1, and negative controls were purchased from Genechem Co. Ltd (Shanghai, China).

\section{Western blotting assays}


For western blotting assays, the tissues and cells were lysed in RIPA protein lysis buffer (Beyotime Institute of Biotechnology, Haimen, China) containing protease inhibitor cocktail and PMSF.

Subsequently, the protein concentrations were measured using a BCA kit (Beyotime Institute of Biotechnology) according to the manufacturer's instructions. A total of $40 \mu \mathrm{g}$ of protein was subjected to SDS-PAGE, which was then separated by gel electrophoresis and transferred to polyvinylidene fluoride (PVDF) membranes. Five percent non-fat dried skim milk was used to block the PVDF membranes for 1 hour at room temperature. Then, the membranes were incubated with primary antibodies overnight at $4^{\circ} \mathrm{C}$. Subsequently, the membranes were washed and incubated in blocking buffer with secondary antibodies for 2 hours at room temperature. Finally, ChemiDoc-XRs+ (Bio-Rad Laboratories, Inc., Hercules, CA, USA) was used to visualize the proteins.

The antibodies used for western blotting were as follows: NUDT1 (Abclonal, A13330), HIF2a (Abclonal, A7553), GAPDH (Proteintech, 60004-1-lg), SOD2 (Abclonal, A1340), HO-1 (Abclonal, A11919), and CAT (Abclonal, A5275).

\section{Cell viability assays}

For cell viability assays, A498 and 786-0 cells were transfected with sh-NC and sh-NUDT1, respectively. Then, the cells were seeded in 96 -well plates at a density of $2 \times 10^{3} /$ well. The proliferation rate of cells was determined using the CCK8 method according to the manufacturer's instructions. Cell viability was assessed at 0, 24, 48, 72 and 96 hours after treatment.

\section{Colony formation assays}

A498 and 786-0 cells transfected with shNUDT1 or the control were plated in 6-well plates at 1000 cells per well. After two weeks, the cells were fixed with methanol and then were stained with $0.05 \%$ crystal violet to enable visualization of the viable colonies (> 50 cells/colonies). The colony forming ability was evaluated from the staining results.

\section{Wound healing assays}

Cells were seeded in 6-well plates. The cells were wound in a straight line by a $10 \mu \mathrm{l}$ pipette tip through the monolayer when the cells reached $70-80 \%$ confluence. Subsequently, the cells were washed with PBS to remove impurities and then were maintained at $37^{\circ} \mathrm{C}$. Images were captured at 0,12 , and 24 hours post wounding.

\section{Transwell assays}

For migration and invasion assays, cells were incubated in serum-free medium for 24 hours. Migration and invasion assays were performed using uncoated and Matrigel ${ }^{\mathrm{T}}{ }^{\mathrm{M}}$-coated Transwell $\circledast$ inserts according to the manufacturer's instructions. Cells were seeded in the top chamber of the insert and then were allowed to invade through the Matrigel. After incubation for 24 hours, cells invading the lower surface of 
the membrane insert were fixed with $100 \%$ methanol. Then, the cells were stained with $0.05 \%$ crystal violet, and 10 regions were randomly selected for counting.

\section{Measurement of intracellular ROS levels}

Cells were stained using a Cell Active Oxygen Detection Kit (Deep Red Fluorescence, Abcam) according to the manufacturer's instructions and then were photographed using a fluorescence microscope.

\section{Cellular MDA measurement}

The method for measuring cell malondialdehyde (MDA) was based on a malondialdehyde (MDA) assay kit (Nanjing Jiancheng Biotechnology Research Institute, Nanjing, China). Intracellular MDA expression was measured using a Multi-Mode Microplate Reader (SpectramMax M5) at $532 \mathrm{~nm}$.

\section{Chromatin immunoprecipitation assay and promoter analysis (ChIP)}

Chromatin immunoprecipitation assay was conducted by SimpleChIP® Enzymatic Chromatin IP Kit (Agarose Beads) \#9002 obtain from CST, which was performed according to the manufacturer's manual. The plasmid with the truncated NUDT1 promoter region was constructed by Tianyi Huiyuan, China. Cell lysates were pretreated with normal rabbit IgG and protein A-agarose. An anti-NUDT1 antibody $(2.0 \mu \mathrm{g})$ was added to the cell lysate, and they were incubated together at $4{ }^{\circ} \mathrm{C}$ overnight. IgG was used as the negative control. The specific primer sets used to amplify the target sequence within the human NUDT1 promoter were designed as follows:

Control Forward 5'- CACCATTGCTAAACCACCCA-3'

Reverse 5'- AGGCTGAGTGGGCATGGG-3'

Site 1 Forward 5'- TGGCCAACATGATGAAACCC-3'

Reverse 5'- GGGTTCAGGCGATTCTCCT-3'

Site 2 Forward 5'- TCTCGAACTCCTGACCTCTG-3'

Reverse 5'- CAGCCTGGATGATAGCAAAACA-3'

Site 3 Forward 5'- CCGGTCTCTATGTCCATCTTTC -3'

Reverse 5'- GAGGGGAAGACAGCGACTC -3'.

\section{Luciferase assays}

The 3' UTR of NUDT1 was constructed into RiboBio (RiboBio, Guangzhou, China), and promoter regions of NUDT1 were constructed by Tianyi Huiyuan, China. Cells were placed in 24-well plates, and complimentary DNA was transfected using Lipofectamine 2000 (Invitrogen) according to the 
manufacturer's instructions. pRL-TK was used as an internal control. The luciferase activity was measured using a double luciferase detection reagent (Promega), and it was performed according to the instructions.

\section{In vivo tumor implantation}

A total of $2 \times 10^{6}$ cells were injected subcutaneously into 6-week-old male nude mice purchased from Vital River Laboratory Animal Technology Co., Ltd. To evaluate the metastatic potential of tumor cells, a nude mouse tail vein metastasis model was used. Tumor growth was measured with a digital caliper every 4 days for 7 weeks. Tumor weight was measured when mice were sacrificed on day 49 after cell implantation. Immunohistochemical staining was conducted using a standard procedure as previously described.

\section{Bioinformatics analysis}

Three independent oxidative stress pathway-related gene sets from the Oncomine database (https://www.oncomine.org) were used for screening. Gene set enrichment analysis (GSEA) was used to assess pathways of enrichment in the gene set. The mRNA levels of genes in normal kidney tissue and ccRCC tissue and relevant information (clinical stage, gender, age, survival time, etc.) of ccRCC patients were obtained from the database of TCGA-KIRC (http://www.cbioportal.org/public-porta).

\section{Statistical analysis}

Data for at least three independent experiments are expressed as the mean \pm SEM. All statistical analyses were performed using t-tests or ANOVA with Excel 2016 (Microsoft) and SPSS Statistics 22.0 (IBM SPSS, Chicago, IL) software. Linear correlation analysis was used to determine the correlation between gene expression levels. Receiver operator characteristic (ROC) curve and area under the curve (AUC) were plotted to detect the best cut-off point and then obtained the highest overall accuracy rate, thereby clarifying different clinical classifications. Pearson correlation coefficient was used to assess the correlation between two factors. $\mathrm{p}<0.05$ was considered statistically significant.

\section{Results}

\section{HIF2 $a$ is related to oxidative stress and its downstream molecule NUDT1 possesses the potential of novel biomarker in ccRCC}

In order to determine whether HIF2a is associated with oxidative stress, gene set enrichment analysis (GSEA) of HIF2a was performed in renal cancer. The results indicated that HIF2a was significantly enriched in the oxidative stress pathway in renal cancer (Figure. 1a). In order to confirm the finding, stable knockdown of HIF2a by lentivirus transfection were completed in A498 and 786-0 cell lines. The western blotting result of cell lines demonstrated that the expression of acknowledged markers of oxidative stress (HO-1, CAT and SOD2) were significantly decreased in the cells with HIF2a knocked down (Figure. 1b)(12, 
28). TCGA-KIRC database analysis results showed that the expression of HO-1 and SOD2 was positively correlated with HIF2a (Figure. S1a). Based on the results of GSEA, western blotting and heatmap visualization, we further confirmed that the biological function of HIF2a in ccRCC was related to oxidative stress pathways. To explore genes downstream of HIF2a that affect oxidative stress, bioinformatics analysis was conducted using the sequencing results of HIF-2a knockdown in renal cancer and oxidative stress pathway-related ccRCC gene datasets (Beroukhim, JONE, GUMZ), which involved the target genes of oxidative stress-related pathway in renal cancer. The overlap of the above gene sets indicated that NUDT1 and SOD2 were dysregulated in CCRCC (Figure. 1c). Furthermore, the analysis of the database from TCGA demonstrated that both NUDT1 and SOD2 were overexpressed in ccRCC (Figure. 1d) (Figure. S1b). The Kaplan-Meier survival analysis showed that cCRCC patients with higher expressions of NUDT1 or SOD2 had a poorer survival while the NUDT1 expression possess more significant differences than SOD2. (Figure. 1e). Moreover, ROC curve analysis also showed that NUDT1 had a larger AUC than SOD2, which meant that there was a better diagnostic value for NUDT1(Figure. 1f). In summary, NUDT1 was the focus of subsequent research.

To confirm the importance of NUDT1 in cCRCC, further bioinformatics analysis strategies were adopted. First, the high expression of NUDT1 in ccRCC was confirmed by using four independent datasets from the Oncomine database (Figure. S1c). At the same time, the expression of NUDT1 was strongly correlated with the clinicopathological parameters of ccRCC (Table 1), and its expression gradually increased with the increase of tumor stage and grade (Figure. S1d). Additionally, analysis of Kaplan-Meier curves by subgroup both for overall and disease-free survival showed that high expression of NUDT1 was highly correlated with poor prognosis in patients with ccRCC (Figured. S2-S3). Univariate and multivariate analyses indicated that NUDT1 is a potential independent prognostic marker for ccRCC (Table 2-3). In addition, ccRCC tissues were analyzed to assess the protein and mRNA levels of NUDT1. The results indicated that the levels of NUDT1 protein and mRNA detected in ccRCC tissues were significantly higher than those in normal tissues (Figure. 1g-i). Compared with the control group, NUDT1 was also highly expressed at the protein and mRNA levels in a ccRCC cell line (Figure. 1j). In general, NUDT1 is highly expressed in ccRCC and associates with poor prognosis of ccRCC. 
Table 1

Correlation between NUDT1 mRNA expression and clinicopathological parameters of cCRCC patients.

\begin{tabular}{|c|c|c|c|c|c|}
\hline \multirow[b]{2}{*}{ Parameter } & & \multicolumn{4}{|c|}{ NUDT1 mRNA expression } \\
\hline & & Number & Low $(n=259)$ & High $(n=260)$ & $P$ value \\
\hline \multirow[t]{2}{*}{ Age(years) } & $<=60$ & 258 & 134 & 124 & \\
\hline & $>60$ & 261 & 125 & 136 & 0.380 \\
\hline \multirow[t]{2}{*}{ gender } & female & 182 & 109 & 73 & \\
\hline & male & 337 & 150 & 187 & $0.001^{*}$ \\
\hline \multirow[t]{2}{*}{ T stage } & $\mathrm{T} 1+\mathrm{T} 2$ & 332 & 196 & 136 & \\
\hline & $\mathrm{T} 3+\mathrm{T} 4$ & 187 & 63 & 124 & $0.000 *$ \\
\hline \multirow[t]{2}{*}{$\mathrm{N}$ stage } & NO + NX & 505 & 257 & 248 & \\
\hline & N1 & 14 & 2 & 12 & $0.006^{*}$ \\
\hline \multirow[t]{2}{*}{ M stage } & $M 0+M X$ & 441 & 237 & 204 & \\
\hline & M1 & 78 & 22 & 56 & $0.000 *$ \\
\hline \multirow[t]{2}{*}{ G stage } & $\mathrm{G} 1+\mathrm{G} 2$ & 239 & 156 & 83 & \\
\hline & $\mathrm{G} 3+\mathrm{G} 4$ & 280 & 103 & 177 & $0.000^{\star}$ \\
\hline \multirow[t]{2}{*}{ TNM stage } & $I+I I$ & 314 & 192 & 122 & \\
\hline & IIII+IV & 205 & 67 & 138 & $0.000 *$ \\
\hline
\end{tabular}


Table 2

Univariate and multivariate analyses of NUDT1 mRNA level and patient overall survival (OS).

\begin{tabular}{|c|c|c|c|c|c|c|}
\hline \multirow[b]{2}{*}{ Variable } & \multicolumn{3}{|c|}{ Univariate analysis } & \multicolumn{3}{|c|}{ Multivariate analysisc } \\
\hline & $\mathrm{HR}^{\mathrm{a}}$ & $95 \% \mathrm{Cl}^{\mathrm{b}}$ & $\mathrm{P}$ & $\mathrm{HR}$ & $95 \% \mathrm{Cl}$ & $P$ \\
\hline \multicolumn{7}{|c|}{ Overall survival $(n=519)$} \\
\hline Age (years) & 1.742 & $1.281-2.367$ & $0.000 *$ & 1.649 & $1.211-2.246$ & $0.002 *$ \\
\hline \multicolumn{7}{|l|}{$\leq 60(n=258)$} \\
\hline \multicolumn{7}{|l|}{$>60(n=261)$} \\
\hline Gender & 0.956 & $0.701-1.303$ & 0.774 & & & \\
\hline \multicolumn{7}{|l|}{ Female $(n=182)$} \\
\hline \multicolumn{7}{|l|}{ Male $(n=337)$} \\
\hline T stage & 3.038 & $2.243-4.114$ & $0.000 *$ & & & \\
\hline \multicolumn{7}{|l|}{ T1 or T2 $(\mathrm{n}=332)$} \\
\hline \multicolumn{7}{|l|}{ T3 or T4 $(n=187)$} \\
\hline $\mathrm{N}$ stage & 3.554 & $1.872-6.747$ & $0.000 *$ & & & \\
\hline \multicolumn{7}{|l|}{ NO or NX $(n=505)$} \\
\hline \multicolumn{7}{|l|}{$N 1(n=14)$} \\
\hline M stage & 4.290 & $3.145-5.854$ & $0.000 *$ & 2.414 & $1.668-3.495$ & $0.000 *$ \\
\hline \multicolumn{7}{|l|}{ M0 or $M X(n=441)$} \\
\hline \multicolumn{7}{|l|}{ M1 $(n=78)$} \\
\hline G grade & 2.607 & $1.855-3.662$ & $0.000^{*}$ & 1.546 & $1.073-2.226$ & $0.019 *$ \\
\hline \multicolumn{7}{|l|}{ G1 or G2 $(n=239)$} \\
\hline \multicolumn{7}{|l|}{ G3 or G4 $(n=280)$} \\
\hline TNM stage & 3.716 & $2.709-5.097$ & $0.000 *$ & 1.927 & $1.303-2.850$ & $0.001 *$ \\
\hline \multicolumn{7}{|l|}{ Stage I + II (n = 314) } \\
\hline Stage III + IV $(n=205)$ & & & & & & \\
\hline
\end{tabular}

a Hazard ratio, estimated from Cox proportional hazard regression model.

b Confidence interval of the estimated HR.

c Multivariate models were adjusted for T, N, M classification, age and gender. 


\begin{tabular}{|c|c|c|c|c|c|c|}
\hline \multirow[b]{2}{*}{ NUDT1 } & \multicolumn{3}{|c|}{ Univariate analysis } & \multicolumn{3}{|c|}{ Multivariate analysisc } \\
\hline & 2.187 & $1.594-2.999$ & $0.000 *$ & 1.547 & $1.114-2.147$ & $0.009 *$ \\
\hline \multicolumn{7}{|l|}{$\operatorname{Low}(n=259)$} \\
\hline \multicolumn{7}{|l|}{$\operatorname{High}(n=260)$} \\
\hline \multicolumn{7}{|c|}{ a Hazard ratio, estimated from Cox proportional hazard regression model. } \\
\hline \multicolumn{7}{|c|}{ b Confidence interval of the estimated HR. } \\
\hline c Multivariate & adjuste & for $\mathrm{T}, \mathrm{N}, \mathrm{M}$ clas & ification, & ge and & yender. & \\
\hline
\end{tabular}


Table 3

Univariate and multivariate analyses of NUDT1 mRNA level and patient disease-free survival (DFS).

\section{Univariate analysis}

\begin{tabular}{llll} 
Variable & $\mathrm{HR}^{\mathrm{a}}$ & $95 \% \mathrm{Cl}^{\mathrm{b}}$ & $\mathrm{P}$ \\
$\begin{array}{l}\text { Disease-free survival }(\mathrm{n}= \\
421)\end{array}$ & & & \\
Age (years) & 1.431 & $0.912-2.246$ & 0.119 \\
$\leq 60(\mathrm{n}=229)$ & & & \\
$>60(\mathrm{n}=192)$ & & & \\
& & & \\
\hline
\end{tabular}

Female $(n=141)$

Male $(\mathrm{n}=280)$

$\begin{array}{llll}\text { T stage } & 6.645 & 4.016- & 0.000 * \\ & & 10.995 & \end{array}$

T1 or T2 $(n=280)$

T3 or T4 $(n=141)$

\begin{tabular}{|c|c|c|c|c|c|c|}
\hline $\mathrm{N}$ stage & \multirow[t]{3}{*}{7.203} & \multirow{3}{*}{$\begin{array}{l}3.429- \\
15.130\end{array}$} & \multirow[t]{3}{*}{$0.000 *$} & \multirow[t]{3}{*}{2.921} & \multirow{3}{*}{$\begin{array}{l}1.378- \\
6.191\end{array}$} & \multirow[t]{3}{*}{$0.005^{\star}$} \\
\hline NO or NX $(n=409)$ & & & & & & \\
\hline$N 1(n=12)$ & & & & & & \\
\hline M stage & \multirow[t]{3}{*}{11.989} & \multirow{3}{*}{$\begin{array}{l}7.595- \\
18.923\end{array}$} & \multirow[t]{3}{*}{$0.000 *$} & \multirow[t]{3}{*}{4.662} & \multirow{3}{*}{$\begin{array}{l}2.802- \\
7.758\end{array}$} & \multirow[t]{3}{*}{$0.000 *$} \\
\hline $\mathrm{M} 0$ or $\mathrm{MX}(\mathrm{n}=368)$ & & & & & & \\
\hline$M 1(n=53)$ & & & & & & \\
\hline G grade & \multirow[t]{3}{*}{5.227} & \multirow[t]{3}{*}{$2.876-9.498$} & \multirow[t]{3}{*}{$0.000 *$} & \multirow[t]{3}{*}{2.966} & \multirow{3}{*}{$\begin{array}{l}1.605- \\
5.481\end{array}$} & \multirow[t]{3}{*}{0.001 * } \\
\hline G1 or G2 $(n=206)$ & & & & & & \\
\hline G3 or G4 $(n=215)$ & & & & & & \\
\hline TNM stage & \multirow[t]{3}{*}{11.115} & \multirow[t]{3}{*}{$\begin{array}{l}6.112- \\
20.215\end{array}$} & \multirow[t]{3}{*}{$0.000^{*}$} & \multirow[t]{3}{*}{4.244} & \multirow[t]{3}{*}{$\begin{array}{l}2.142- \\
8.409\end{array}$} & \multirow[t]{3}{*}{$0.000 *$} \\
\hline Stage I + II $(n=267)$ & & & & & & \\
\hline Stage III + IV $(n=154)$ & & & & & & \\
\hline
\end{tabular}

a Hazard ratio, estimated from Cox proportional hazard regression model.

b Confidence interval of the estimated HR.

c Multivariate models were adjusted for T, N, M classification, age and gender. 

NUDT1
3.252
$1.934-5.468$
$0.000^{*}$

$\operatorname{Low}(\mathrm{n}=210)$

High $(n=211)$

a Hazard ratio, estimated from Cox proportional hazard regression model.

b Confidence interval of the estimated HR.

c Multivariate models were adjusted for T, N, M classification, age and gender.

\section{Nudt1 Promotes The Progression Of Ccrcc}

Based on the above results, we speculated that the function of NUDT1 involves a potential impact on the progression of ccRCC. To verify this hypothesis, we constructed A498 and 786-0 cell lines with NUDT1 knockdown or overexpression by lentivirus or plasmid transfection, respectively (Figure. 2a-b). In order to clarify the proliferation effect of knocking down NUDT1 in ccRCC cells, colony formation was conducted. The results showed that the proliferation ability of A498 and 786-0 cells was significantly inhibited by NUDT1 knock down (Figure. 2c). The CCK8 assays were used to detect the proliferation of ccRCC cells after knockdown or overexpression of NUDT1. The results demonstrated that knockdown of NUDT1 in the ccRCC cell lines significantly decreased the proliferation rate of ccRCC cells (Figure. 2d). Consistently, NUDT1 overexpression increased the proliferation rate in ccRCC cells (Figure. 2e). Migration and invasion are also important indicators of tumor progression. Transwell and wound healing assays were performed to investigate the effect of NUDT1 expression on migration and invasion ability in ccRCC cells. In terms of these abilities, knockdown of NUDT1 inhibited the migration and invasion of ccRCC cells (Figure. 2f, Figure. S4), while overexpression of NUDT1 enhanced the migration and invasion in ccRCC cells (Figure. $2 \mathrm{~g}$ ). Taken together, the results above demonstrated that the expression of NUDT1 played a vital role in cell proliferation and cell migration/invasion in ccRCC cell lines. The overexpression of NUDT1 could promote the cell growth and metastasis, while NUDT1 knock down would inhibit these abilities in ccRCC cell lines.

\section{NUDT1 is correlated with oxidative stress and involved in key biological processes in ccRCC}

The GSEA analysis focusing on NUDT1 expression in CCRCC was performed and the results showed that NUDT1 was highly related to renal cell carcinoma, cell metabolism, and energy production (Figure. 3a), which were all basic biological functions in RCC. To explore the funtions of NUDT1 in CCRCC, we further performed full transcriptome sequencing (RNA-seq) on ccRCC cell lines with NUDT1 knocked down. Based on the bioinformatics analysis of gene sequencing results, differentially expressed genes were identified between samples and analyzed. The results demonstrated that the expression of oxidative stress markers (HO-1 and CAT) were significantly decreased in the cells with NUDT1 knocked down 
(Figure. 3b-d). After mapping the transcripts to a gene ontology (GO) database, we found that NUDT1 played a key role in cell development and antioxidant activity pathways (Figure. 3e). Kyoto Encyclopedia of Genes and Genomes (KEGG) pathway enrichment analysis was performed to further analyze the effect of NUDT1 in ccRCC. The results showed that the differentially expressed genes were mainly enriched in basic processes of cell activity, which indicated that NUDTI played a vitral role in ccRCC cells (Fig. 3f-g). Therefore, we conclude that NUDT1 is highly correlated with oxidative stress and involved in key biological processes in ccRhCC.

\section{Nudt1 Inhibits Oxidative Stress In Ccrcc}

CcRCC is a special tumor type that exhibits a significant change in cellular redox balance (29). As we discussed above, the results indicated the functions of NUDT1 in ccRCC cells, but the mechanism of NUDT1 in ccRCC remains unclear. To explore the relationship between NUDT1 and oxidative stress in cCRCC, bioinformatics analyses were performed. We analyzed the database from TCGA-KIRC by GSEA. The results indicated that NUDT1 participated in mitochondrial formation and division in CCRCC, further demonstrating the effect of NUDT1 on cellular oxidative stress (Fig. 4a). To prove the connection between NUDT1 and oxidative stress, we generated a correlation heatmap and linear correlation curves between NUDT1 and the most critical ROS level-related molecules HO-1 and SOD2 $(30,31)$ based on data from the database from TCGA-KIRC. The results showed that NUDT1 was significantly positively correlated with molecules related to oxidative stress (Figure. $4 b-c$ ). Subsequently, we detected the expression of oxidative stress markers HO-1, CAT, and SOD2 in ccRCC cell lines with NUDT1 stable knock down or overexpression. The results indicated that HO-1, CAT, and SOD2 protein levels were significantly downregulated in stably silenced NUDT1 cell lines, and these protein levels were significantly upregulated in cell lines stably overexpressing NUDT1 (Figure. 4d-e). To further understand the effect of NUDT1 on the oxidative stress environment of ccRCC cells, we used ROS Deep Red Dye and MDA assays to determine the level of oxidative stress in the above cell lines. The ROS Deep Red Dye showed that knockdown of NUDT1 could increase ROS levels, while overexpression of NUDT1 could inhibit ROS levels (Figure. 4f-g) in cCRCC cell lines. With the MDA assay, we found that knockdown of NUDT1 enhanced the relative MDA level in both A498 and 786-0 cells, while overexpression of NUDT1 significantly decreased the relative MDA level (Figure. 4h-i). This means that knockdown of NUDT1 can significantly increase the level of oxidative stress in cCRCC cells, while overexpression of NUDT1 can significantly reduce the level of oxidative stress in ccRCC cells.

\section{The oxidative stress pathway promoted by NUDT1 knockdown plays an important role in the carcinogenesis of HIF2 $a$ in ccRCC}

Since NUDT1 is a downstream molecule that was identified after HIF2a silencing and the oxidative stress pathway activated by NUDT1 plays an important role in cCRCC, we hypothesize that the enhanced cellular oxidative stress caused by the knockdown of NUDT1 might play an important role in the carcinogenic effect of HIF2 $a$ in ccRCC. To confirm and evaluate the role of NUDT1 in the HIF2a pathway, we conducted 
a functional response experiment. The ccRCC cell lines knocked down HIF2 $a$ and knocked down NUDT1 was constructed using a lentivirus capable of silencing NUDT1 in a ccRCC cell line stably silencing HIF2a (Figure. 5a). Cell viability assays showed that HIF2a knockdown decreased cell growth while NUDT1 overexpression enhanced cell growth in renal cells. Interestingly, overexpression of NUDT1 reversed cell growth inhibition in cells with HIF2a knockdown (Figure. 5b). Furthermore, the western blotting assays we performed demonstrated that the expression of NUDT1 and HIF2a could regulate the expression of HO-1, CAT and SOD2 in cCRCC (Figure. 5c). Similar to the cell proliferation ability, the transwell assays performed with similarly transfected ccRCC cells indicated consistent results. Knockdown of HIF2a decreased cell migration and invasion, while overexpression of NUDT1 enhanced cell migration and invasion. Consistently, the inhibition of cell migration and invasion in CcRCC caused by HIF2a knockdown could be reversed via overexpression of NUDT1 (Figure. 5d). The ROS and oxidative stress levels in ccRCC cells were shown by ROS Deep Red dye. The results showed that ROS levels in HIF2a knockdown cell lines were significantly increased, while NUDT1 overexpression significantly reduced the effect of HIF2a knockdown on cCRCC ROS levels (Figure. 5e). In summary, These data confirms that the oxidative stress caused by NUDT1 knockdown plays an important role in the carcinogenesis of HIF2a.

\section{HIF2 $a$ regulates NUDT1 expression by directly binding to its promoter region}

Above, we verified that NUDT1 is an important downstream target of HIF2a. Based on the premise that HIF2 $a$ is an important oncogenic gene of cCRCC, we speculate that NUDT1 be directly regulated by HIF2a transcription. We analyzed HIF2a-related pathways by GSEA. The results showed that HIF2a was involved in the process of cellular oxidative stress (Figure. 1a) and nucleoside metabolism (Figure. 6a), which is consistent with the function of NUDT1 in cells. Therefore, the specific mechanism of HIF2a regulating NUDT1 has become the focus of our attention. HIF2a, as one of the most important oncogenes in ccRCC, predominantly acts as a transcription factor that directly promotes the expression of downstream genes. Transcriptome sequencing after HIF2a knockdown, western blotting and qPCR showed that the expression of NUDT1 at the protein and RNA levels decreased with HIF2a silencing, indicating that there is a positive regulatory relationship between HIF2 $a$ and NUDT1 (Figure. 6b-d). Therefore, we first explored the regulatory mechanism of HIF2a on NUDT1 from the perspective of direct transcription. Based on the HIF2 $a$ binding sequence (32), we identified three potential binding sites in the 3000 bp region upstream of the NUDT1 promoter: sites 1, 2 and 3 (Figure. S5). Chromatin immunoprecipitation (ChIP) assays were used to verify the binding of HIF2 $a$ to these potential sites. We found that HIF2a binds to all predicted potential hypoxic response elements (HREs) of NUDT1 in A498 and 786-0 cell lines (Figure. 6e, Figure. S5). A luciferase reporter gene assay was introduced to further illustrate the specific roles of the sites. The truncated plasmids were constructed based on the binding sites of the NUDT1 promoter region HIF2a as site1, site2 and site3, respectively. We found that the reduction of HIF2a silencing on luciferase activity was significantly reversed after site 1 was excised, while removal of sites 2 or 3 had no significant significance, which suggested that site 1 was the main site of HIF2a regulation of NUDT1 (Figure. 6f). Finally, we conclude that HIF2 $a$ acts as a transcription factor to directly increase NUDT1 expression by binding to HIF2a response elements in the NUDT1 promoter region. 


\section{Knockdown Of Nudt1 Suppresses Ccrcc Progression In Vivo}

Encouraged by the in vitro results, in vivo experiments based on NUDT1 were carried out. A498 cells with NUDT1 stably silenced (shNUDT1-1 and shNUDT1-2) were injected subcutaneously into the axilla of immune-deficient mice to generate a xenograft tumor model. The mice were observed for 7 weeks, and the tumor sizes were measured every week. Consistent with the in vitro experiments, knockdown of NUDT1 inhibited tumor growth based on tumor weight and size (Figure. 7a-c). Meanwhile, an immune deficiency mouse tail vein metastasis model was employed to assess the metastatic ability of the tumor cells. After 7 weeks of observation, we observed that knockdown of NUDT1 significantly reduced ccRCC liver metastasis (Figure. 7d). Therefore, to further evaluate the effect of NUDT1 on ccRCC metastasis, fluorescence images were obtained of living mice. The results showed that the whole-body fluorescence intensity of mice was significantly reduced after NUDT1 silencing, which indicated that silencing NUDT1 could significantly reduce ccRCC metastasis (Figure. 7e).

In vitro experiments confirmed that silencing NUDT1 could significantly promote oxidative stress in ccRCC. Therefore, we also evaluated changes in oxidative stress levels in vivo. Through immunohistochemistry (IHC), we found that after silencing NUDT1, the oxidative stress markers HO-1, CAT, and SOD2 were significantly reduced, and Ki67, a measure of tumor malignancy, was also significantly reduced (Figure. 7f). The above results demonstrated that silencing NUDT1 in vivo can also significantly promoted oxidative stress and inhibited the renal tumor progression.

Based on this evidence, we propose a model in which HIF2a acts as a transcription factor to directly increases NUDT1 expression by binding to the HIF2a response element in the NUDT1 promoter. NUDT1 can reduce the level of oxidative stress and balance the mitochondrial metabolism in tumor cells, thereby promoting ccRCC progression. When HIF2 $a$ is knocked down, it can target the reduction of NUDT1 expression, leading to increased levels of ROS and oxidative stress in tumor cells, thereby inhibiting the occurrence and progression of ccRCC (Figure. $7 \mathrm{~g}$ ).

\section{Discussion}

Recently, an increasing number of studies have shown that oxidative stress plays an important role in cCRCC. Widespread activation of HIF2a is an important feature of ccRCC. Relevant literature has confirmed that HIF2a affects the progression of oxidative stress (33), but the specific mechanism linking HIF2 $a$ and oxidative stress has not yet been clarified. In this study, we identified a novel pathway by which HIF2a regulates oxidative stress levels in tumor cells through NUDT1. Mechanistic investigations have shown that a large amount of ROS is produced in highly metabolized malignant tumors, leading to the destruction of cell structure and inhibiting tumor progression (34). HIF2a can directly regulate the expression of NUDT1 to eliminate the effect of ROS on tumor cells, leading to the progression of ccRCC. 
Oxidative stress is characterized as an imbalance between the production of cellular oxidants and the process of removing their byproducts (8). Reactive oxygen species (ROS) can be used as indicators of oxidative stress, which is mainly produced in the mitochondrial electron transport chain during cell metabolism and plays a vital role in normal cell signaling pathways such as proliferation and apoptosis $(35,36)$. However, excessive ROS levels can cause structural damage in cells $(34)$. Highly metabolically active cancer cells can actually produce more ROS than normal cells, and these metabolically active cells consequently showed more evidence of DNA damage and buffer system engagement (7). Therefore, ccRCC needs some "means" of reducing ROS damage while promoting cell proliferation and survival (37).

Increased levels of ROS in tumor cells result in the accumulation of high levels of 8-oxo-dGTP in their nucleotide pools (38-40). NUDT1 can eliminate excessive 8-oxo-dGTP to avoid oxidative damage of tumor cell nucleic acid (41). Recent studies have also shown that NUDT1 can reduce the level of ROS induced by oncogenic RAS, and plays an important role in oncogenic RAS-mediated transformation and proliferation $(20,42,43)$. Overexpression of NUDT1 may have several protective functions for cancer cells by hydrolyzing 8-oxo-dGTP or reducing ROS levels. Based on the protective properties of NUDT1 against oxidative stress in cancer cells, NUDT1 inhibitors have been developed as potential anticancer drugs (38, 44). HIF2 $a$ is a common transcription factor, and its role of HIF2 $a$ in ccRCC has been confirmed. The relationship between HIF2 $a$ and oxidative stress has been mentioned in the current research $(45,46)$, but its mechanistic link is still unclear. In this study, we not only revealed a new pathway by which HIF2a can regulate oxidative stress but also demonstrated a new mechanism by which it can regulate oxidative stress in ccRCC. Typically, the activation of HIF2a in ccRCC cells directly promotes the expression of NUDT1, thereby reducing the level of oxidative stress in the cells to eliminate ROS effects. In summary, this promotion process may be one of the important mechanisms by which HIF2a eliminates the effects of ROS and promotes the ccRCC process.

As an important oncogene in ccRCC, HIF2a has been widely studied and treated in ccRCC. Currently, most of the research has focused on the role of HIF2 $a$ in angiogenesis in cCRCC. The first-line drug sunitinib that is used to treat ccRCC also targets downstream molecules of HIF2a, specifically vascular endothelial growth factor receptor (VEGFR) and platelet-derived growth factor receptors (PDGFR)(47, 48). However, $10-20 \%$ of advanced RCC patients are inherently unresponsive to sunitinib treatment, and most of the remaining patients eventually develop resistance and tumor progression after 6-15 months of therapy (49). Thus, it is urgent to further clarify the mechanism of HIF2a cancer promotion and find new strategies for the treatment of ccRCC. According to recent research, oxidative stress plays a key role in tumorigenesis and development. A large amount of evidence proves that the pro-cancer effect of HIF2a on cCRCC is not only related to angiogenesis but that oxidative stress is also a factor that cannot be ignored(33). Nevertheless, there have been few reports that have assessed targeted oxidative stress therapy for ccRCC. This study confirmed that NUDT1 is a key molecule by which HIF2a regulates oxidative stress in cCRCC. Based on these results, targeted NUDT1 therapy may play an important role in oxidative stress in ccRCC. Therefore, we propose a new drug combination strategy: a combination therapy of anti-angiogenesis and NUDT1 targeting inhibitors. 


\section{Conclusion}

Through comprehensive analysis, this study identified a new pathway by which HIF2a can directly regulate NUDT1 expression in cCRCC. NUDT1 can eliminate the killing effect of oxidative stress on ccRCC by reducing ROS. In addition, this study provides new insights into the role of HIF2a in regulating the cCRCC process. These findings provide us with a strategy for overcoming cellular oxidative stress defense in the treatment of cCRCC and present certain guiding significance for the development of new drugs and combined drug treatment strategies for ccRCC therapy.

\section{Abbreviations}

NUDT1

Nucleoside diphosphate linked moiety X-type motif 1

HIF2a

Hypoxia-inducible factor 2alpha ccRCC:clear cell renal cell carcinoma

RCC

Renal cell carcinoma

$\mathrm{VHL}$

The von Hippel-Lindau ROS:reactive oxygen species

SOD2

Superoxide dismutase 2

HO-1

Heme oxygenase 1

CAT

Catalase

GSEA

Gene set enrichment analysis

$\mathrm{ROC}$

Receiver operator characteristic

AUC

Area under the curve

IHC

Immunohistochemical

CHIP

Chromatin immunoprecipitation assay

\section{Declarations}

Acknowledgements

Not applicable. 


\section{Funding}

This work was supported by the National Natural Science Foundation of China (Grant No. 81672528, 81672524), Hubei Provincial Natural Science Foundation of China (2018CFA038), application project of the Wuhan Science and Technology Bureau (2016060101010053), Independent Innovation Foundation of Huazhong University of Science and Technology (118530309), Clinical Research Physician Program of Tongji Medical College, Huazhong University of Science and Technology (5001530015) and Integrated Innovation Team for Major Human Disease Program of Tongji Medical College, Huazhong University of Science and Technology.

\section{Authors' Contributions}

JS, ZYX, KSW and CFY carried out experiments. YH and WX took on the statistical analysis. HGL, TBX and HMY gave assistance in collecting tissue samples or animal experiments. HMY made revision to the language. KC and XPZ conceived experiments and analyzed data. All authors were involved in writing the paper and had final approval of the submitted and published versions.

\section{Availability of Data and Materials}

The analyzed datasets generated during the current study are available from the corresponding author on reasonable request.

\section{Ethics declarations}

\section{Ethics approval and consent to participate}

All human studies were reviewed and approved by the Institutional Review Board (IRB) of Union Hospital, Tongji Medical College, Huazhong University of Science and Technology, and written informed consent was provided according to the World Medical Association Declaration of Helsinki.

\section{Consent for publication}

Consent to publish has been obtained from all authors.

\section{Competing interests}

The authors declare that they have no competing interests.

\section{References}

1. Sarfaty M, Leshno M, Gordon N, Moore A, Neiman V, Rosenbaum E, et al. Cost Effectiveness of Nivolumab in Advanced Renal Cell Carcinoma. Eur Urol. 2018;73(4):628-34.

2. Qiu B, Ackerman D, Sanchez DJ, Li B, Ochocki JD, Grazioli A, et al. HIF2alpha-Dependent Lipid Storage Promotes Endoplasmic Reticulum Homeostasis in Clear-Cell Renal Cell Carcinoma. Cancer 
discovery. 2015;5(6):652-67.

3. Martinez-Saez O, Gajate Borau P, Alonso-Gordoa T, Molina-Cerrillo J, Grande E. Targeting HIF-2 alpha in clear cell renal cell carcinoma: A promising therapeutic strategy. Crit Rev Oncol Hematol. 2017;111:117-23.

4. Keith B, Johnson RS, Simon MC. HIF1alpha and HIF2alpha: sibling rivalry in hypoxic tumour growth and progression. Nature reviews Cancer. 2011;12(1):9-22.

5. Shen C, Beroukhim R, Schumacher SE, Zhou J, Chang M, Signoretti S, et al. Genetic and functional studies implicate HIF1alpha as a 14q kidney cancer suppressor gene. Cancer discovery. 2011;1(3):222-35.

6. Purdue MP, Johansson M, Zelenika D, Toro JR, Scelo G, Moore LE, et al. Genome-wide association study of renal cell carcinoma identifies two susceptibility loci on 2p21 and 11q13.3. Nat Genet. 2011;43(1):60-5.

7. Abu Aboud O, Habib SL, Trott J, Stewart B, Liang S, Chaudhari AJ, et al. Glutamine Addiction in Kidney Cancer Suppresses Oxidative Stress and Can Be Exploited for Real-Time Imaging. Cancer research. 2017;77(23):6746-58.

8. Day AM, Brown JD, Taylor SR, Rand JD, Morgan BA, Veal EA. Inactivation of a peroxiredoxin by hydrogen peroxide is critical for thioredoxin-mediated repair of oxidized proteins and cell survival. Molecular cell. 2012;45(3):398-408.

9. Schieber M, Chandel NS. ROS function in redox signaling and oxidative stress. Current biology: CB. 2014;24(10):R453-62.

10. Cairns RA, Harris IS, Mak TW. Regulation of cancer cell metabolism. Nature reviews Cancer. 2011;11(2):85-95.

11. Sena LA, Chandel NS. Physiological roles of mitochondrial reactive oxygen species. Molecular cell. 2012;48(2):158-67.

12. Gorrini C, Harris IS, Mak TW. Modulation of oxidative stress as an anticancer strategy. Nat Rev Drug Discovery. 2013;12(12):931-47.

13. Hu CJ, Wang LY, Chodosh LA, Keith B, Simon MC. Differential roles of hypoxia-inducible factor 1alpha (HIF-1alpha) and HIF-2alpha in hypoxic gene regulation. Molecular cellular biology. 2003;23(24):9361-74.

14. Gruber M, Hu CJ, Johnson RS, Brown EJ, Keith B, Simon MC. Acute postnatal ablation of Hif-2alpha results in anemia. Proc Natl Acad Sci USA. 2007;104(7):2301-6.

15. Scortegagna M, Ding K, Oktay Y, Gaur A, Thurmond F, Yan LJ, et al. Multiple organ pathology, metabolic abnormalities and impaired homeostasis of reactive oxygen species in Epas1-/- mice. Nat Genet. 2003;35(4):331-40.

16. Sakumi K, Furuichi M, Tsuzuki T, Kakuma T, Kawabata S, Maki H, et al. Cloning and expression of cDNA for a human enzyme that hydrolyzes 8-oxo-dGTP, a mutagenic substrate for DNA synthesis. J Biol Chem. 1993;268(31):23524-30. 
17. Maki $H$, Sekiguchi M. MutT protein specifically hydrolyses a potent mutagenic substrate for DNA synthesis. Nature. 1992;355(6357):273-5.

18. Weinberg F, Hamanaka R, Wheaton WW, Weinberg S, Joseph J, Lopez M, et al. Mitochondrial metabolism and ROS generation are essential for Kras-mediated tumorigenicity. Proc Natl Acad Sci USA. 2010;107(19):8788-93.

19. Grabocka E, Commisso C, Bar-Sagi D. Molecular pathways: targeting the dependence of mutant RAS cancers on the DNA damage response. Clinical cancer research: an official journal of the American Association for Cancer Research. 2015;21(6):1243-7.

20. Patel A, Burton DG, Halvorsen K, Balkan W, Reiner T, Perez-Stable C, et al. MutT Homolog 1 (MTH1) maintains multiple KRAS-driven pro-malignant pathways. Oncogene. 2015;34(20):2586-96.

21. Rai P, Onder TT, Young JJ, McFaline JL, Pang B, Dedon PC, et al. Continuous elimination of oxidized nucleotides is necessary to prevent rapid onset of cellular senescence. Proc Natl Acad Sci USA. 2009;106(1):169-74.

22. Rai P, Young JJ, Burton DG, Giribaldi MG, Onder TT, Weinberg RA. Enhanced elimination of oxidized guanine nucleotides inhibits oncogenic RAS-induced DNA damage and premature senescence. Oncogene. 2011;30(12):1489-96.

23. Gad H, Koolmeister T, Jemth AS, Eshtad S, Jacques SA, Strom CE, et al. MTH1 inhibition eradicates cancer by preventing sanitation of the dNTP pool. Nature. 2014;508(7495):215-21.

24. Fujishita T, Okamoto T, Akamine T, Takamori S, Takada K, Katsura M, et al. Association of MTH1 expression with the tumor malignant potential and poor prognosis in patients with resected lung cancer. Lung cancer (Amsterdam Netherlands). 2017;109:52-7.

25. Li J, Yang CC, Tian XY, Li YX, Cui J, Chen Z, et al. MutT-related proteins are novel progression and prognostic markers for colorectal cancer. Oncotarget. 2017;8(62):105714-26.

26. Akiyama S, Saeki H, Nakashima Y, limori M, Kitao H, Oki E, et al. Prognostic impact of MutT homolog1 expression on esophageal squamous cell carcinoma. Cancer medicine. 2017;6(1):258-66.

27. Pudelko L, Rouhi P, Sanjiv K, Gad H, Kalderen C, Hoglund A, et al. Glioblastoma and glioblastoma stem cells are dependent on functional MTH1. Oncotarget. 2017;8(49):84671-84.

28. Glorieux C, Calderon PB. Catalase, a remarkable enzyme: targeting the oldest antioxidant enzyme to find a new cancer treatment approach. Biological chemistry. 2017;398(10):1095-108.

29. Pelicano H, Carney D, Huang P. ROS stress in cancer cells and therapeutic implications. Drug resistance updates: reviews commentaries in antimicrobial anticancer chemotherapy. 2004;7(2):97110.

30. Ren J, Su D, Li L, Cai H, Zhang M, Zhai J, et al. Anti-inflammatory effects of Aureusidin in LPSstimulated RAW264.7 macrophages via suppressing NF-kappaB and activating ROS- and MAPKsdependent Nrf2/HO-1 signaling pathways. Toxicol Appl Pharmcol. 2020;387:114846.

31. Kim H, Lee YD, Kim HJ, Lee ZH, Kim HH. SOD2 and Sirt3 Control Osteoclastogenesis by Regulating Mitochondrial ROS. Journal of bone mineral research: the official journal of the American Society for Bone Mineral Research. 2017;32(2):397-406. 
32. Mastrogiannaki M, Matak P, Keith B, Simon MC, Vaulont S, Peyssonnaux C. HIF-2alpha, but not HIF1alpha, promotes iron absorption in mice. J Clin Investig. 2009;119(5):1159-66.

33. Tormos KV, Chandel NS. Inter-connection between mitochondria and HIFs. J Cell Mol Med. 2010;14(4):795-804.

34. Herraiz C, Crosas-Molist E, Sanz-Moreno V. Reactive oxygen species and tumor dissemination: Allies no longer. Molecular cellular oncology. 2016;3(2):e1127313.

35. Finkel T. Redox-dependent signal transduction. FEBS Lett. 2000;476(1-2):52-4.

36. Jabs T. Reactive oxygen intermediates as mediators of programmed cell death in plants and animals. Biochem Pharmacol. 1999;57(3):231-45.

37. Chandel NS, Tuveson DA. The promise and perils of antioxidants for cancer patients. N Engl J Med. 2014;371(2):177-8.

38. Gad H, Koolmeister T, Jemth AS, Eshtad S, Jacques SA, Ström CE, et al. MTH1 inhibition eradicates cancer by preventing sanitation of the dNTP pool. Nature. 2014;508(7495):215-21.

39. Borrego S, Vazquez A, Dasí F, Cerdá C, Iradi A, Tormos C, et al. Oxidative Stress and DNA Damage in Human Gastric Carcinoma: 8-0xo-7'8-dihydro-2'-deoxyguanosine (8-oxo-dG) as a Possible Tumor Marker. Int J Mol Sci. 2013;14(2):3467-86.

40. lida T, Furuta A, Kawashima M, Nishida J, Nakabeppu Y, Iwaki T. Accumulation of 8-oxo-2'deoxyguanosine and increased expression of hMTH1 protein in brain tumors. Neuro Oncol. 2001;3(2):73-81.

41. Kennedy CH, Pass HI, Mitchell JB. Expression of human MutT homologue (hMTH1) protein in primary non-small-cell lung carcinomas and histologically normal surrounding tissue. Free Radic Biol Med. 2003;34(11):1447-57.

42. Giribaldi MG, Munoz A, Halvorsen K, Patel A, Rai P. MTH1 expression is required for effective transformation by oncogenic HRAS. Oncotarget. 2015;6(13):11519-29.

43. Rai P. Human Mut T, Homolog. 1 (MTH1): a roadblock for the tumor-suppressive effects of oncogenic RAS-induced ROS. Small GTPases. 2012;3(2):120-5.

44. Huber KV, Salah E, Radic B, Gridling M, Elkins JM, Stukalov A, et al. Stereospecific targeting of MTH1 by (S)-crizotinib as an anticancer strategy. Nature. 2014;508(7495):222-7.

45. Cao MQ, You AB, Cui W, Zhang S, Guo ZG, Chen L, et al. Cross talk between oxidative stress and hypoxia via thioredoxin and HIF-2a drives metastasis of hepatocellular carcinoma. FASEB journal: official publication of the Federation of American Societies for Experimental Biology. 2020;34(4):5892-905.

46. Saint-Martin A, Martínez-Ríos J, Castañeda-Patlán MC, Sarabia-Sánchez MA, Tejeda-Muñoz N, Chinney-Herrera A, et al. Functional Interaction of Hypoxia-Inducible Factor 2-Alpha and Autophagy Mediates Drug Resistance in Colon Cancer Cells. Cancers (Basel). 2019;11(6).

47. Gnarra JR, Tory K, Weng Y, Schmidt L, Wei MH, Li H, et al. Mutations of the VHL tumour suppressor gene in renal carcinoma. Nat Genet. 1994;7(1):85-90. 
48. Faivre S, Demetri G, Sargent W, Raymond E. Molecular basis for sunitinib efficacy and future clinical development. Nature reviews Drug discovery. 2007;6(9):734-45.

49. Molina AM, Lin X, Korytowsky B, Matczak E, Lechuga MJ, Wiltshire R, et al. Sunitinib objective response in metastatic renal cell carcinoma: analysis of 1059 patients treated on clinical trials. Eur $\mathrm{J}$ Cancer. 2014;50(2):351-8.

\section{Figures}




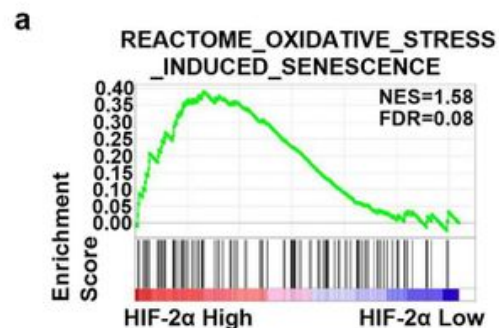

c ShHIF-2 $\alpha$ Beroukhim JONES sequencing 4 GUMZ

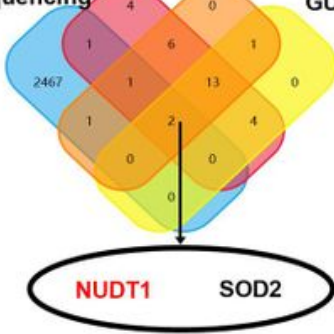

e

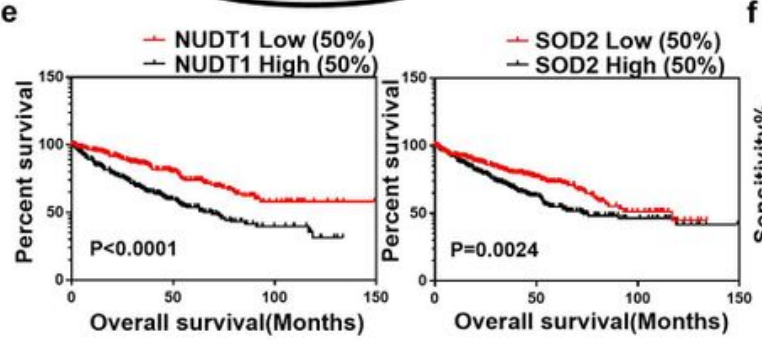

g

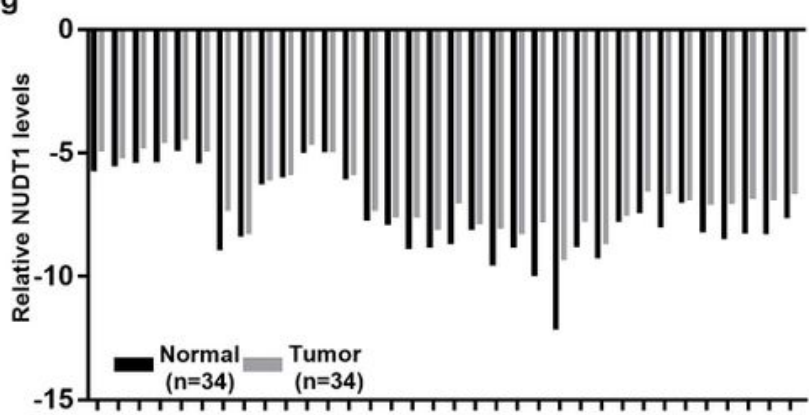

b

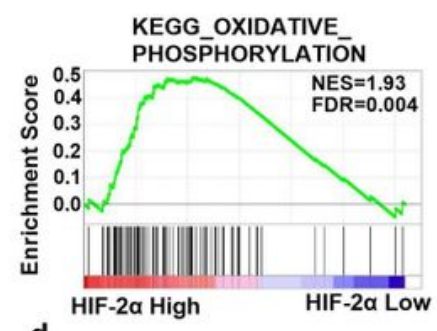

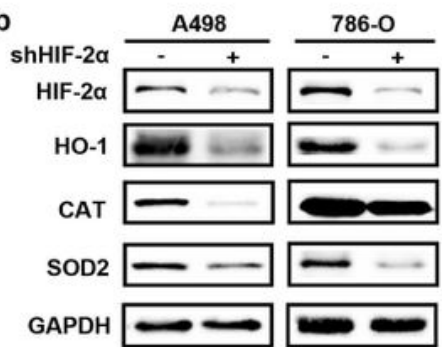

TCGA(ccRCC, $n=533)$

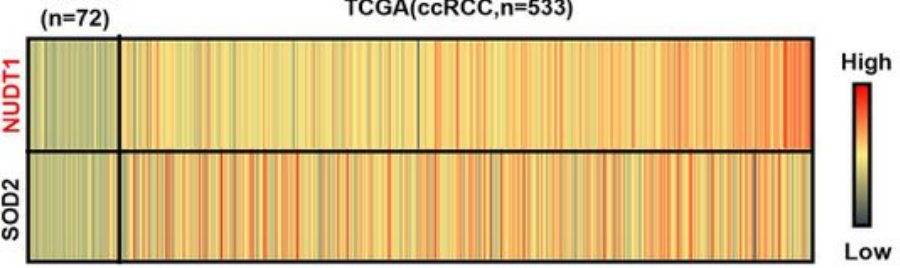

i

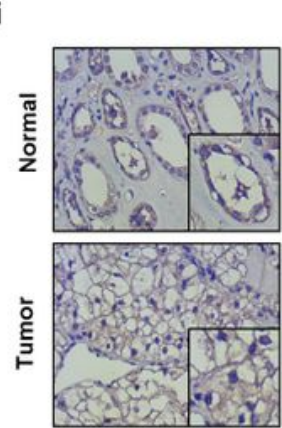

Clear cell renal cell carcinoma

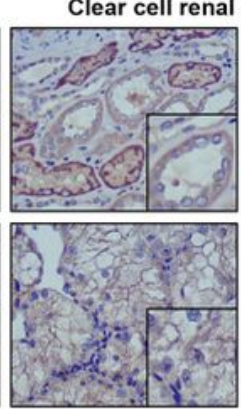

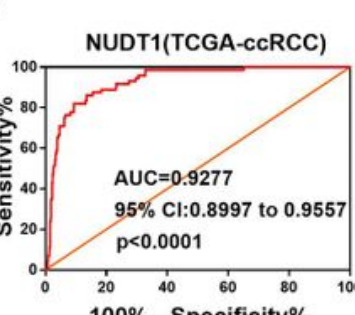

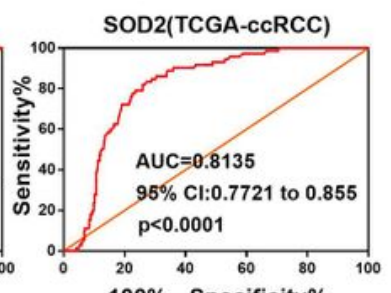

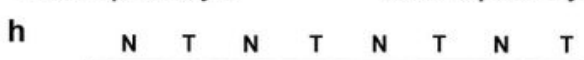

NUDT1 $\rightarrow$ C
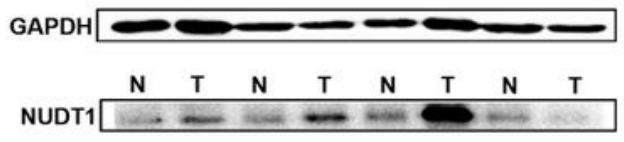

GAPDH $\longrightarrow-\infty-\infty$

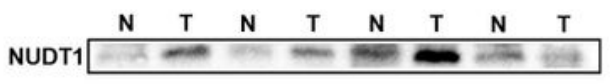

GAPDH
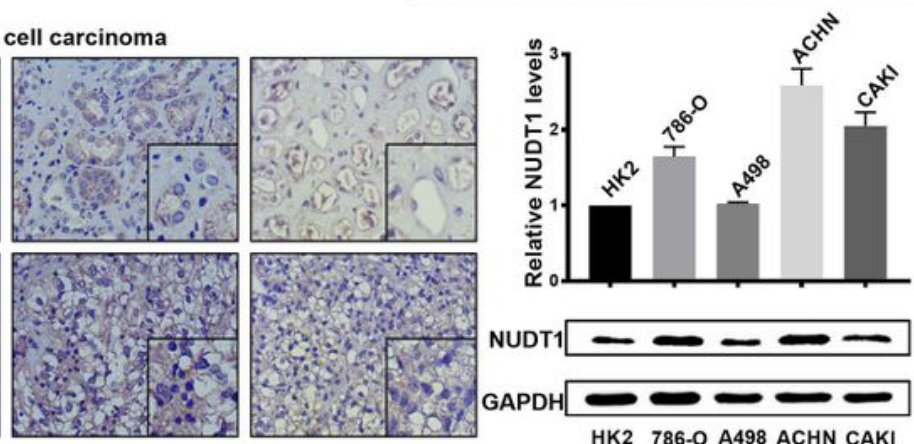

NUDT1 $-\longrightarrow$

GAPDH $\longrightarrow-\infty-\infty$

HK2 786-O A498 ACHN CAKI

\section{Figure 1}

HIF2 $a$ is related to oxidative stress and its downstream molecule NUDT1 is overexpressed and indicates poor survival in ccRCC. (a) GSEA correlation charts were screened according to the database from TCGA$\mathrm{KIRC}$, and they reveal the correlation between oxidative stress and HIF-2a mRNA levels in ccRCC. FDR $<25 \%$ and $P<0.05$ was considered statistically significant. (b) The protein levels of oxidative stressrelated marker genes HO-1, CAT, and SOD2 in HIF-2a knockout cell lines are shown by western blotting. (c) 
A Venn diagram composed of three independent oxidative stress pathway-related gene sets from the Oncomine database (https://www.oncomine.org) and the entire transcriptome sequencing data obtained after stable HIF2a knockdown. The data were screened in ccRCC. (d) The heatmap of NUDT1 and SOD2 mRNA levels in 533 ccRCC tissues and 72 matched tissues in TCGA database. (e) Kaplan-Meier curves of NUDT1 and SOD2 expression in cCRCC patients were used to measure the impact of overall survival (OS). (f) The ROC curves for NUDT1 (AUC=0.9277 95\% Cl: 0.8997 to 0.9557; $P<0.0001$ ) and SOD2 (AUC=0.8135 $95 \% \mathrm{Cl}: 0.7721$ to $0.855 ; \mathrm{P}<0.0001)$ in ccRCC. (g) Levels of NUDT1 mRNA in 34 pairs of ccRCC tissues and adjacent nonmalignant tissues; t-test, $P<0.0001$. (h) Levels of the NUDT1 protein in ccRCC tissues and adjacent nonmalignant tissues. (i) Immunohistochemical (IHC) staining for NUDT1 in ccRCC tissues and adjacent nonmalignant tissues. (j) NUDT1 protein and mRNA levels in $5 \mathrm{ccRCC}$ cell lines and normal cell lines; t-test, ${ }^{*} \mathrm{P}<0.05$, $* \star * \mathrm{P}<0.001$ and ${ }^{\star \star \star *} \mathrm{P}<0.0001$. 

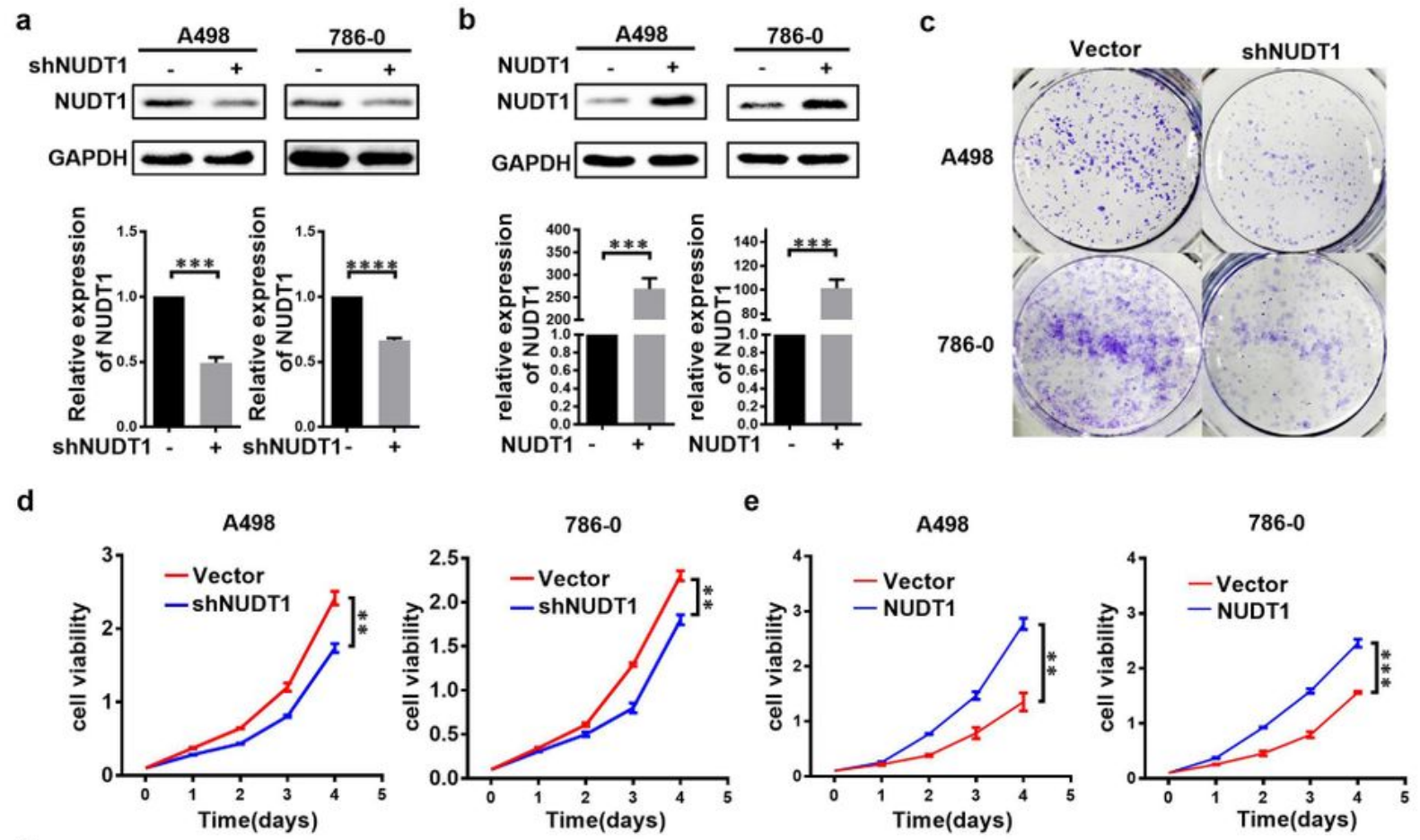

f
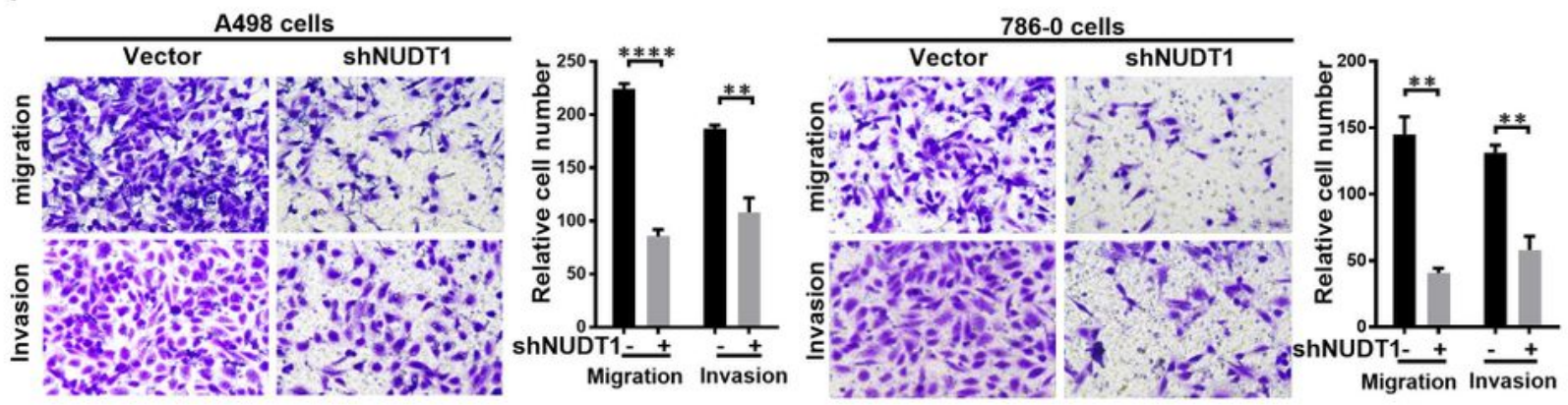

g
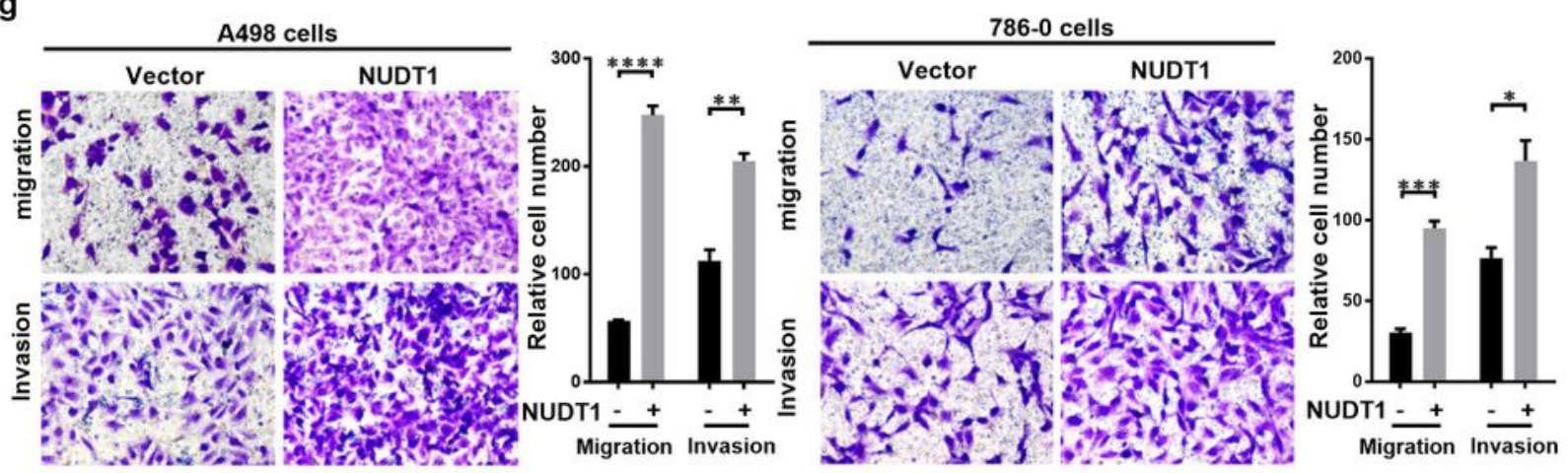

\section{Figure 2}

NUDT1 promotes the progression of cCRCC. NUDT1 knockdown or overexpressing ccRCC cell lines were constructed by transfecting siRNA and an overexpression plasmid, respectively. The results are expressed as the mean \pm SEM of three independent experiments, and there are at least three replicates in each

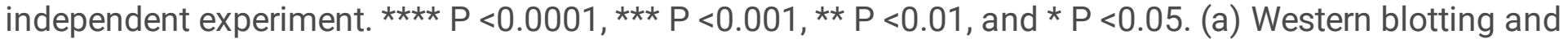
qPCR were used to verify NUDT1 knockdown at the protein and mRNA levels, respectively. (b) Western 
blotting and qPCR were used to verify NUDT1 overexpression at the protein and mRNA levels, respectively. (c) Colony formation experiment results are shown for NUDT1 knockdown cells. (d) CCK8 assays were used to determine the cell growth of NUDT1 knockdown cell lines. (e) CCK8 were used assays to determine the cell growth curve of NUDT1-overexpressing cell lines. (f) The results of the transwell assay of the migration and invasion of NUDT1 knockdown cell lines are shown. (g) The results of the transwell assay of the migration and invasion of NUDT1-overexpressing cell lines. $* \star \star \star ~ P<0.0001$, *** $\mathrm{P}<0.001$, ** $\mathrm{P}<0.01$, and * $\mathrm{P}<0.05$.

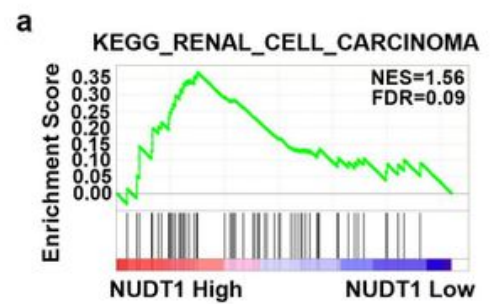

b
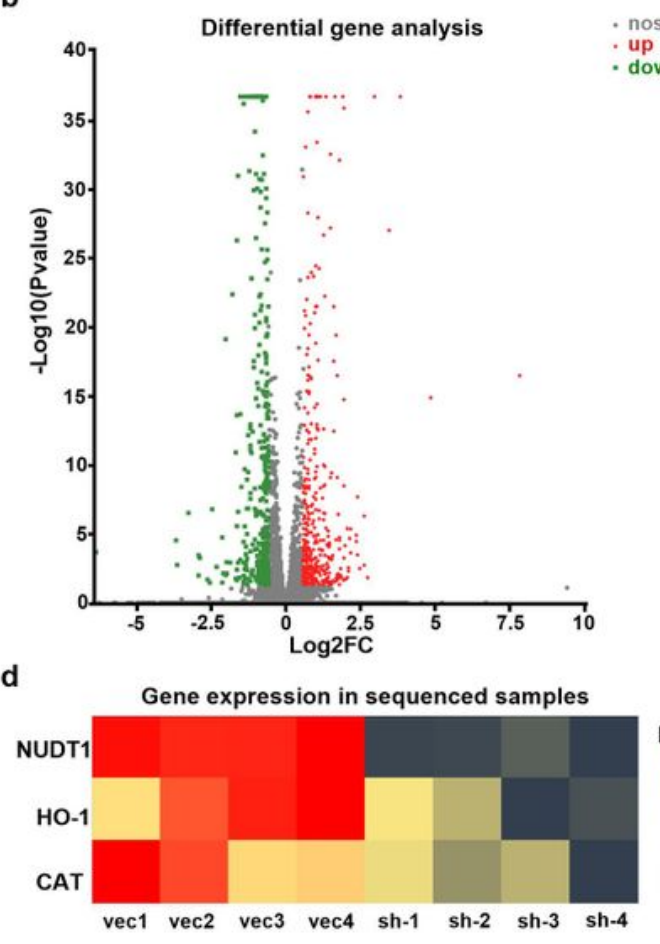

f

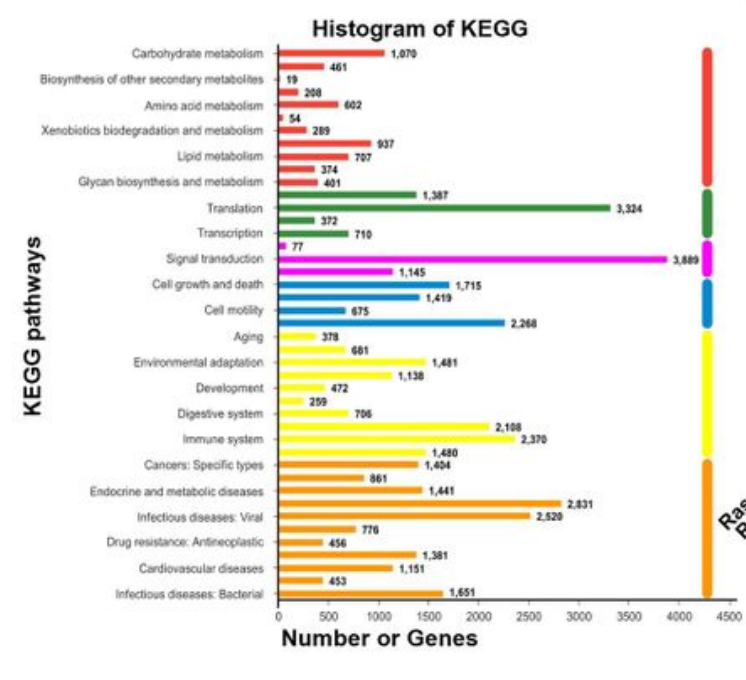

c

e
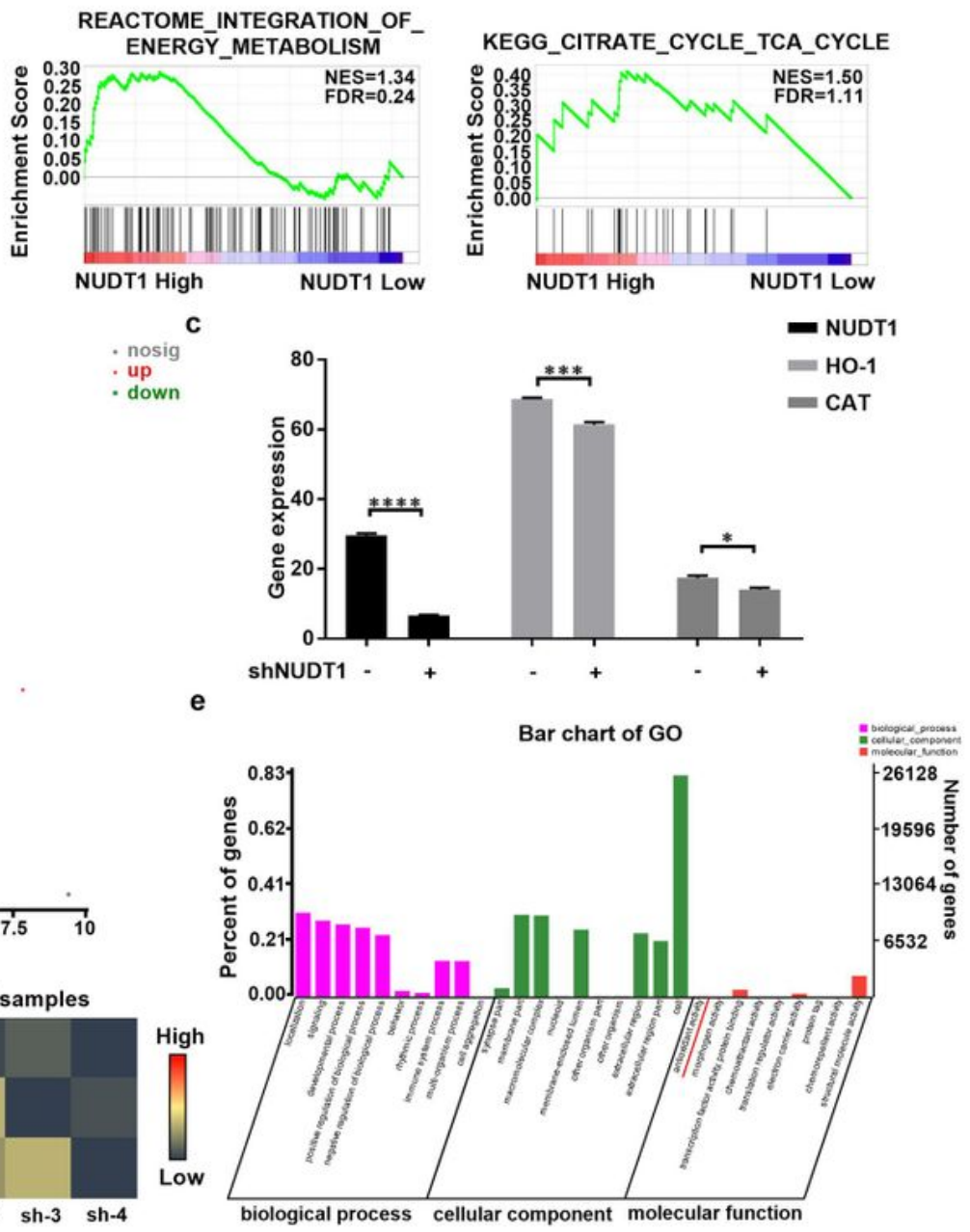

g

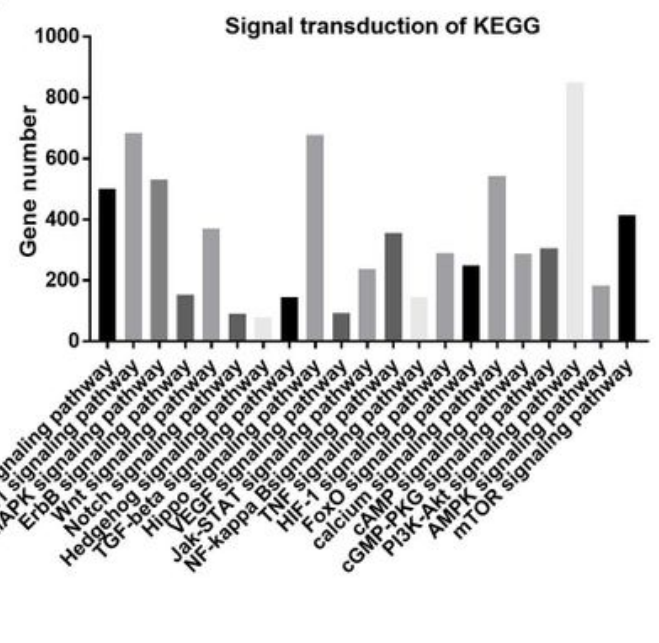




\section{Figure 3}

NUDT1 is correlated with oxidative stress and involved in key biological processes in ccRCC. A498 cell samples transfected with vector and shNUDT1 were subjected to full transcriptome sequencing (RNASeq) to quantify changes in gene expression and analyze the transcriptome after gene knockout. (a) GSEA correlation charts were screened according to the database from TCGA-KIRC, reflecting the correlation between genes related to basic cell functions and NUDT1 mRNA levels in ccRCC. FDR $<25 \%$ and $\mathrm{P}<0.05$ was considered statistically significant. (b) DESeq2 software was used to analyze the expression differences of the sequenced samples, and the results are shown plotted as volcanic maps. FC (fold difference of this gene between samples) $>1.5, p<0.05$. (c-d) Histogram and heatmap and histogram based on the gene expression levels of NUDT1, HO-1, and CAT in the sequenced samples. (e) Graphs that map transcripts to the gene ontology (GO) database are shown, and they enabled pathway analysis. (f-g) Graphs that map transcripts to the Kyoto Encyclopedia of Genes and Genomes (KEGG) database are shown, and they enabled pathway analysis. 
a

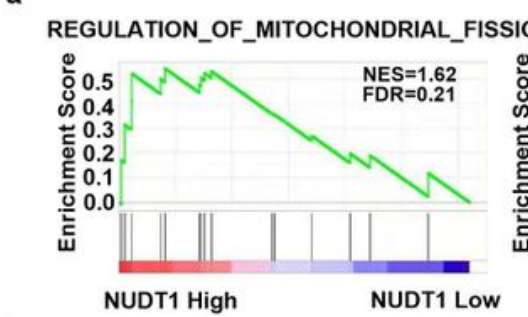

b

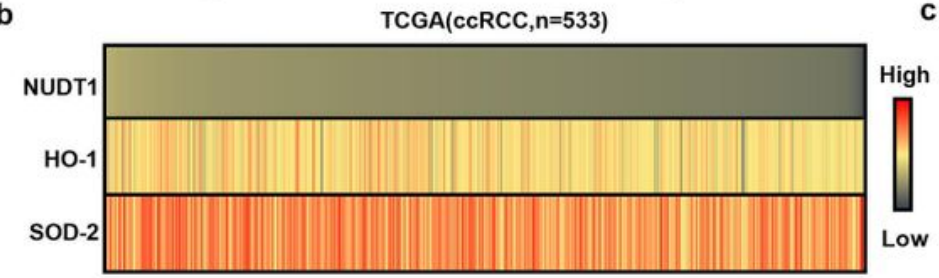

REACTOME_TRANSCRIPTIONAL_ACTIVATION _OF_MITOCHONDRIAL_BIOGENESIS
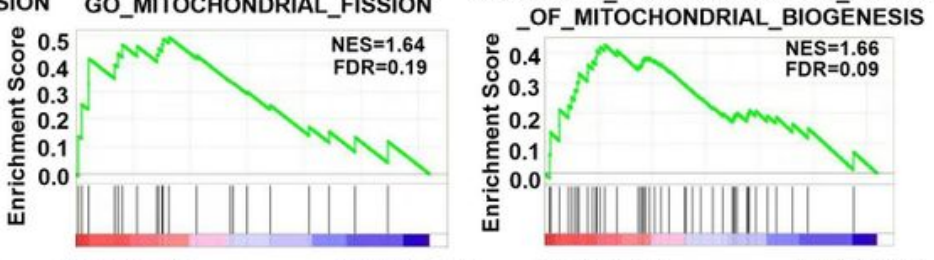

NUDT1 High

NUDT1 Low

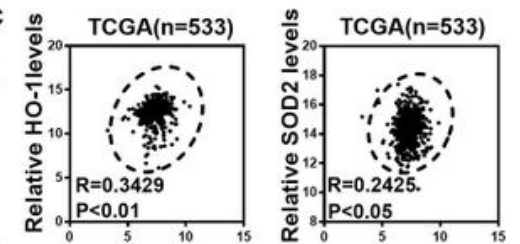

Relative NUDT1 levels Relative NUDT1 levels

d
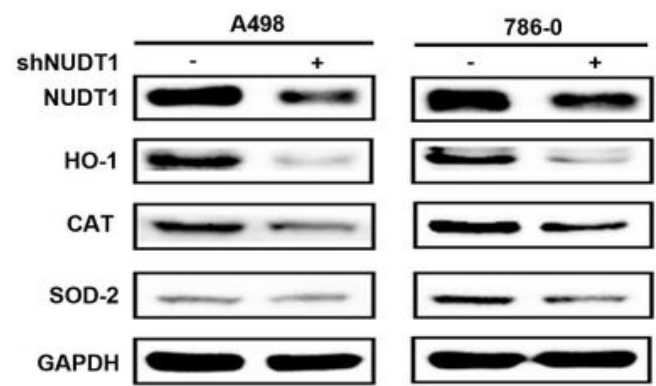

f
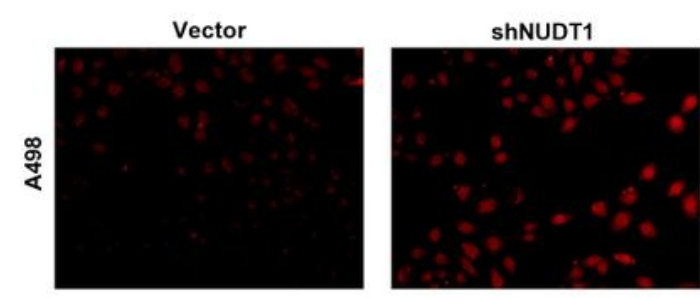

e

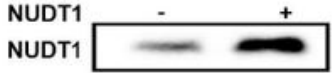

HO-1 rever

СAT $\longrightarrow-\longrightarrow$

SOD-2 $\longrightarrow$

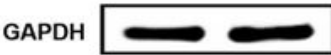

g
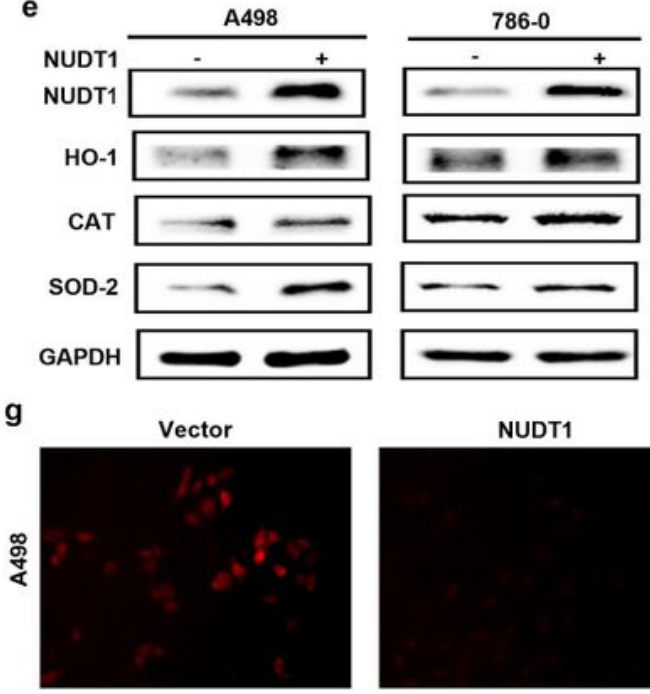

NUDT1
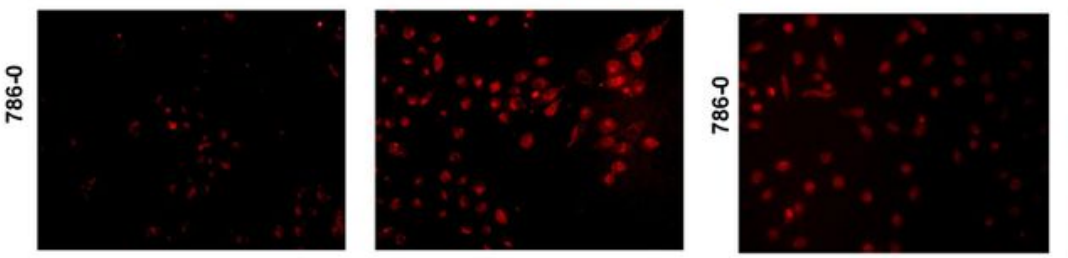

i

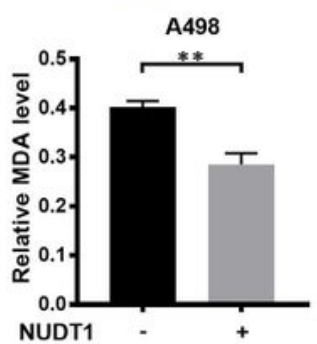

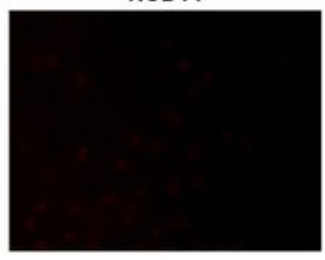
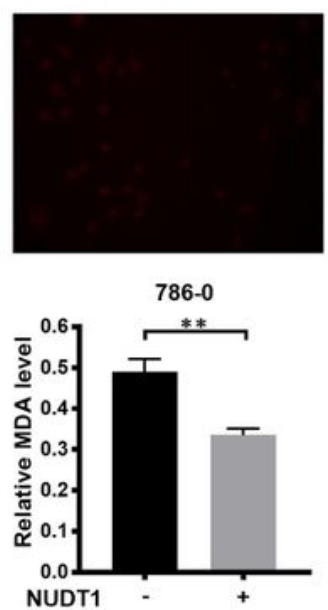

\section{Figure 4}

NUDT1 inhibits oxidative stress in CCRCC. (a) The GSEA correlation charts were screened according to the TCGA-KIRC database, reflecting the correlation between mitochondrial production and NUDT1 mRNA levels in ccRCC. FDR $<25 \%$ and $\mathrm{P}<0.05$ was considered statistically significant. (b-c) The correlation heatmap and the linear correlation curve between NUDT1 and the most critical molecules related to lipid browning (HO-1 and SOD2) based on the data from the TCGA-KIRC database. (d-e) Protein levels of ROS 
and oxidative stress-related marker genes (HO-1, CAT, and SOD2) in NUDT1 knockdown and overexpressing cell lines were detected by western blotting. (f-g) Images of NUDT1 knockdown and overexpressing cell lines stained with a Cellular Reactive Oxygen Species Detection Assay Kit (Deep Red Fluorescence) (ab186029) in a black wall/clear bottom 96-well plate. (h) MDA content in NUDT1 knockdown and overexpressing cell lines was measured. Experiments were repeated 3 times, as described in the Materials and Methods section. $* \star \star * ~ P<0.0001$, *** $P<0.001$, ** $\mathrm{P}<0.01$, and $* \mathrm{P}<0.05$.

a

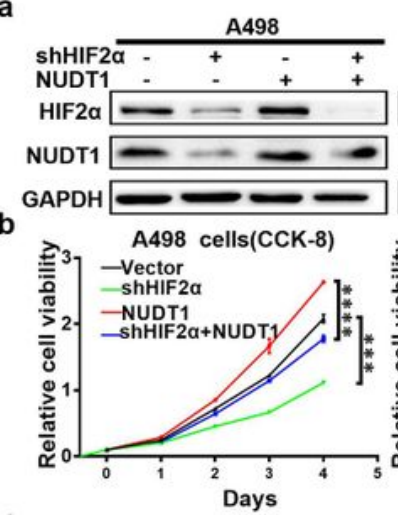

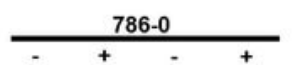
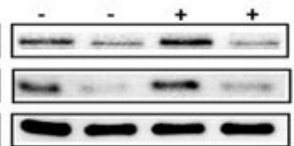

786-0 cells(CCK-8)

$\left.\begin{array}{l}2.5 \\ 2.0\end{array}\right]$ - - - shHIF $2 \alpha$

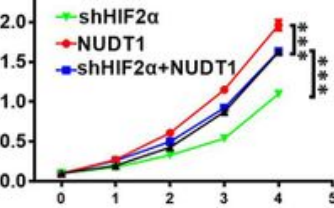

Days
C

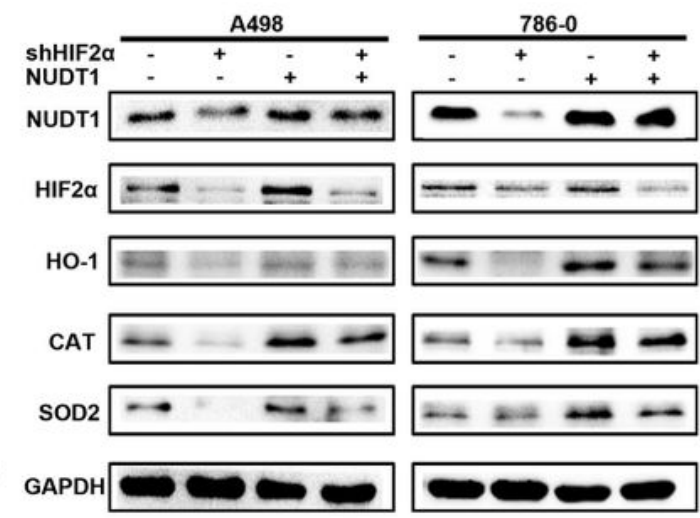

d A498 cells

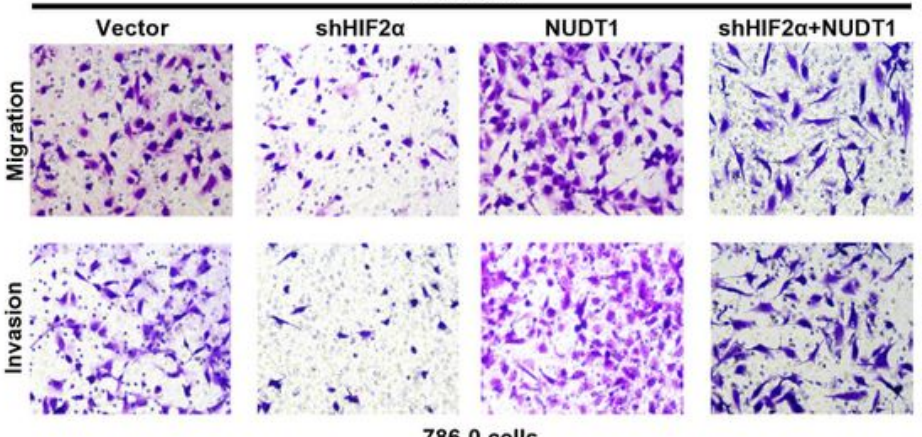

786- 0 cells

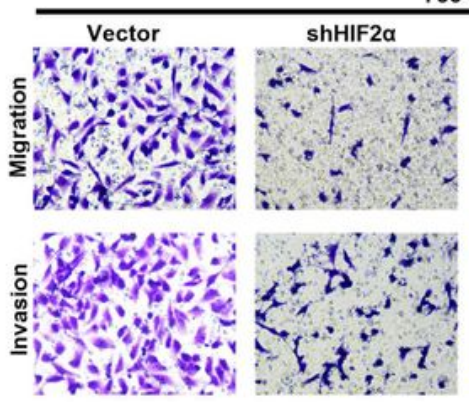
noorn

shHIF2 $\alpha+$ NUDT1
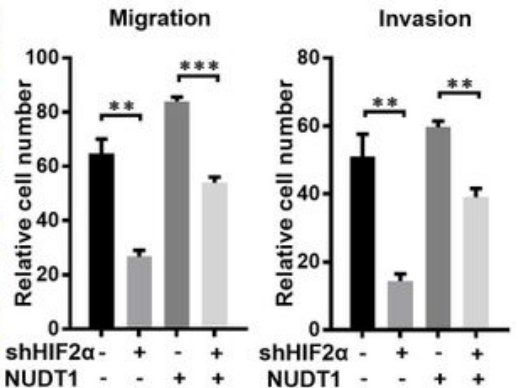

NUDT1 - - + + NUDT1
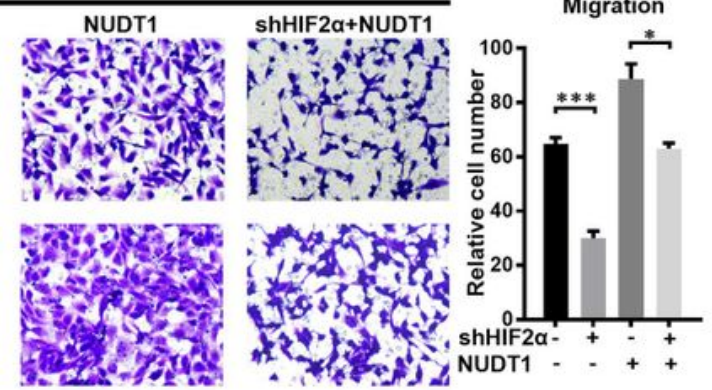

Invasion
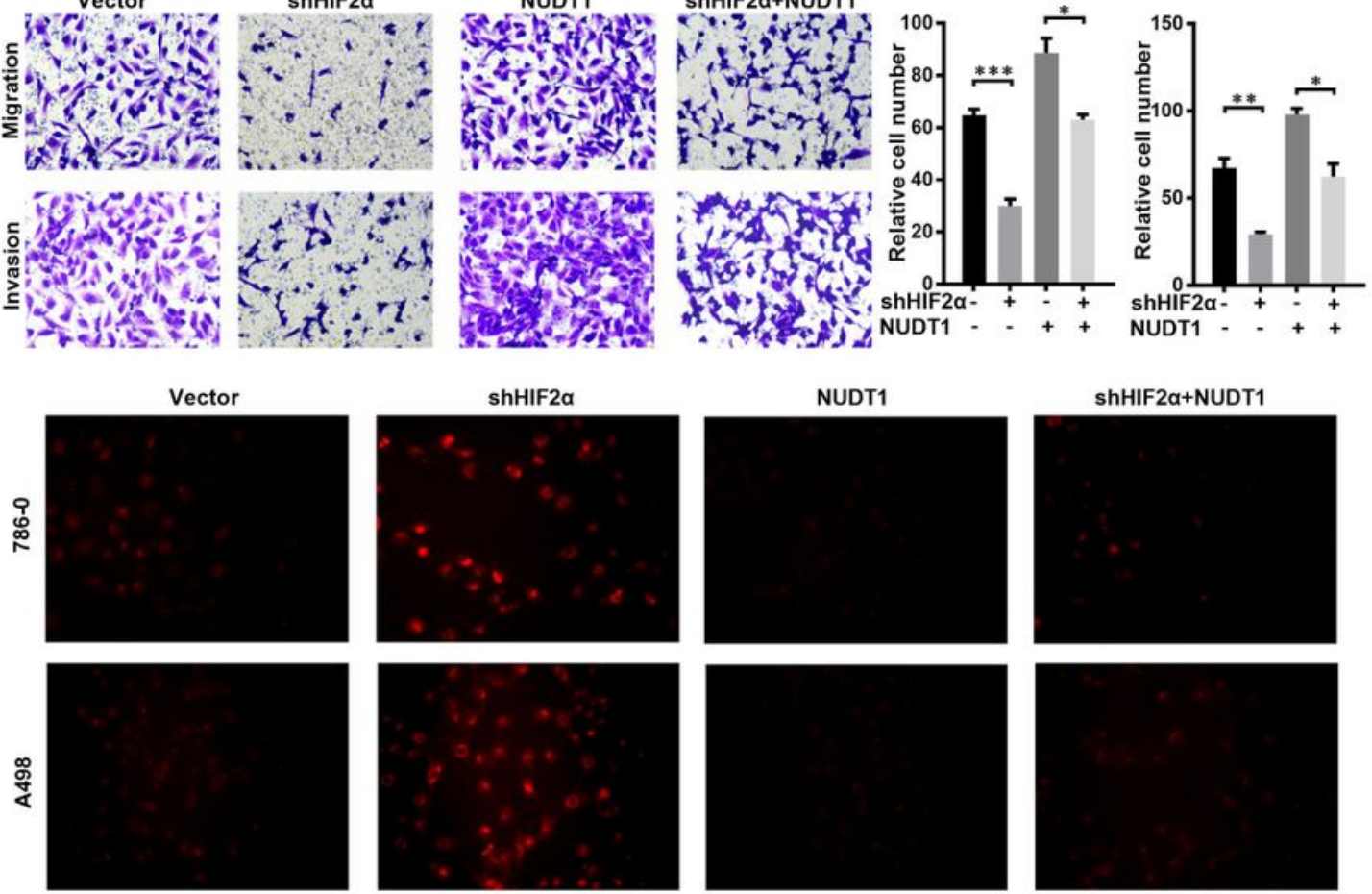

Figure 5 
The oxidative stress pathway promoted by NUDT1 knockdown plays an important role in the carcinogenesis of HIF2a in cCRCC. We performed double transfection in A498 and 786-0 cells with ShHIF2 $a$ and NUDT1 overexpression plasmids to cause HIF2 $a$ knockdown and NUDT1 overexpression. (a) HIF-2a and NUDT1 protein levels in transfected cell lines are shown by western blotting. (b) Cell growth curves based on CCK8 assays are shown for transfected cell lines. ${ }^{*} * * P<0.0001,{ }^{*} * *<0.001$, and $\star \star P<0.01$. (c) Western bolt showing levels of the HIF2a, NUDT1, HO-1, CAT, SOD2 in the indicated cell lines. (d) The results of the transwell assay of the migration and invasion of transfected cell lines. $* \star \star * P$ $<0.0001$, $* * * P<0.001$, $* * P<0.01$, and $* P<0.05$. (e) Images of NUDT1-transfected cell lines stained with the Cellular Reactive Oxygen Species Detection Assay Kit in a black wall/clear bottom 96-well plate. 
a

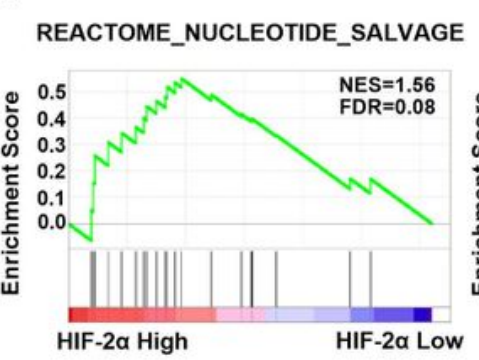

c

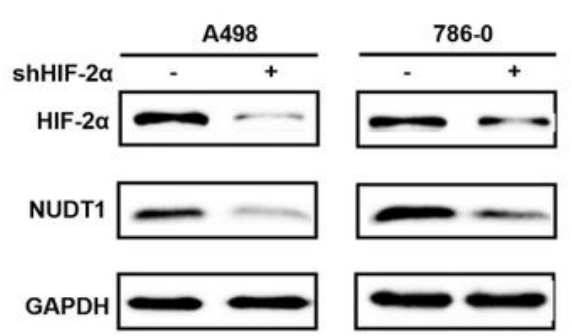

e

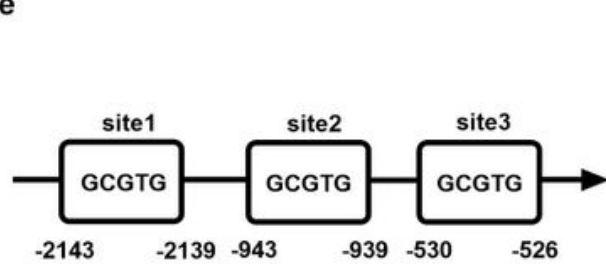

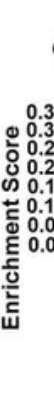

GO_NUCLEOSIDE_BISPHOSPHATE METABOLIC PROCESS

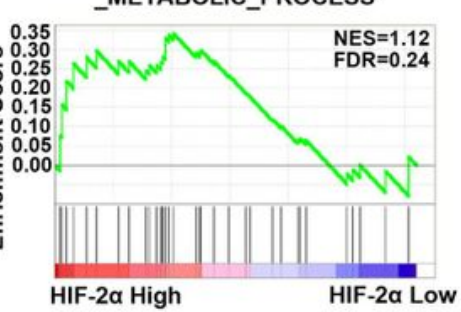

d b

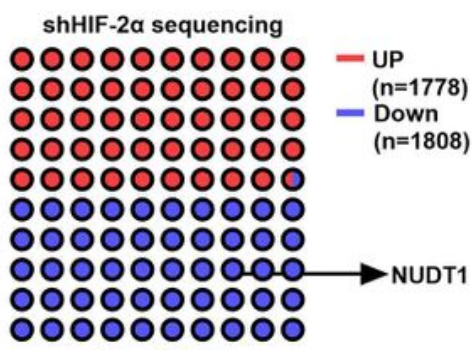

Total $=3586$

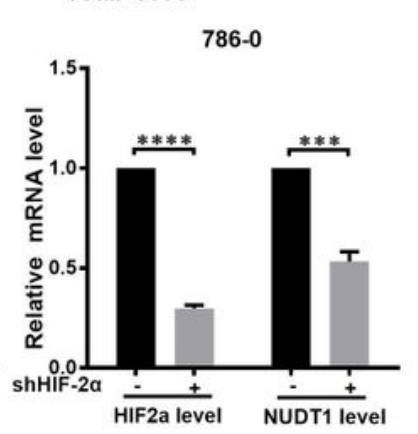

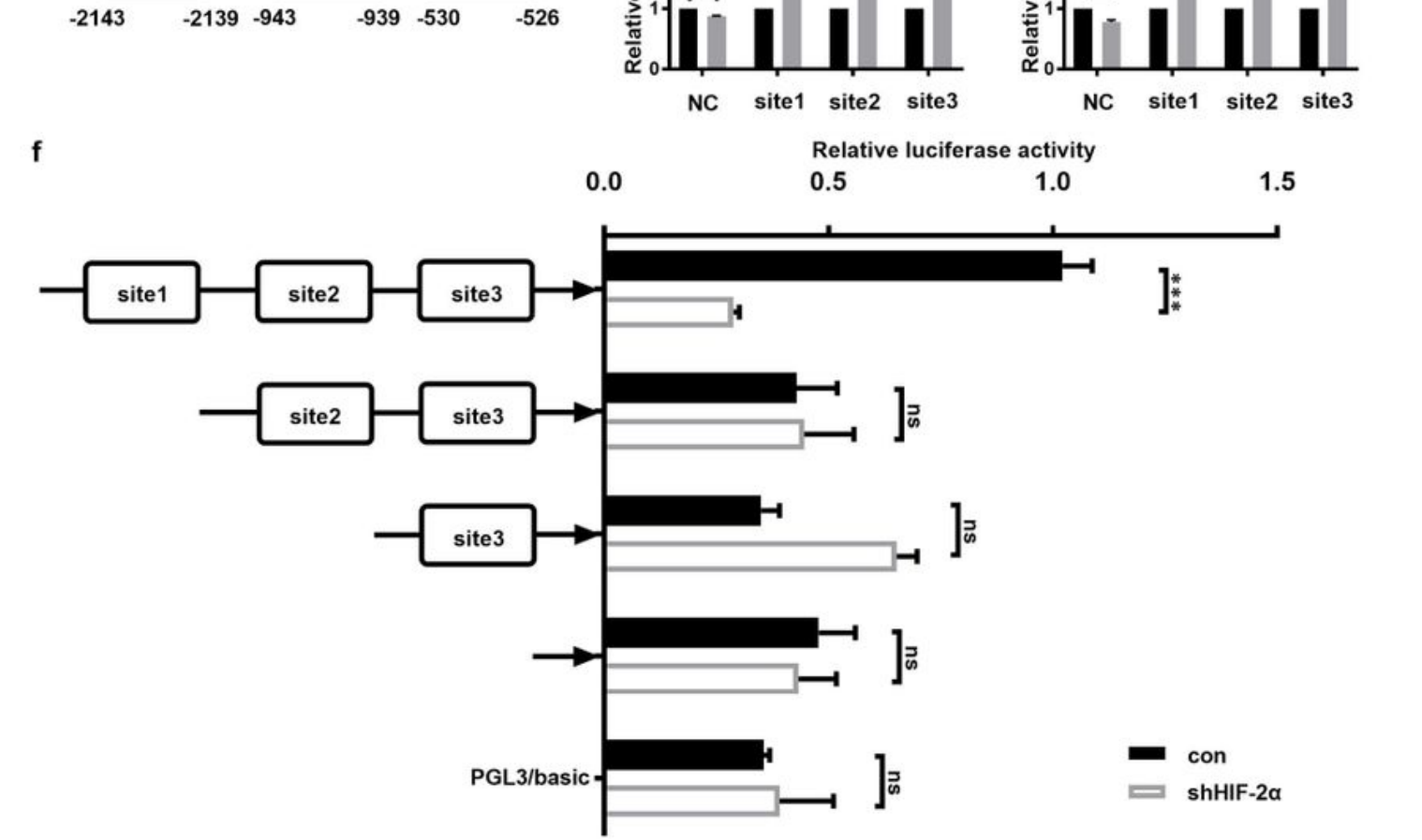

\section{Figure 6}

HIF2a regulates NUDT1 expression by directly binding to its promoter region. (a) GSEA correlation charts were screened according to the database from TCGA-KIRC, reflecting the correlation between nucleoside metabolism and HIF-2a mRNA levels in cCRCC. FDR $<25 \%$ and $\mathrm{P}<0.05$ was considered statistically significant. (b) Sequencing data for the expression of NUDT1 in the entire transcriptome after stable knockout of HIF2a. (c-d) The protein and mRNA levels of NUDT1 after knocking down HIF2a are shown 
by western blotting and qPCR. (e) ChIP experiment results of potential HIF2 binding sites in the NUDT1 promoter are based on the HIF2a binding sequence. ( $f$ ) The results of the luciferase assay were obtained according to the materials and methods described previously. The truncation of the promoter showed that HIF2a bound to the NUDT1 promoter 1 region (-2143 to -2139$)$, which is important for HIF2a to regulate NUDT1.
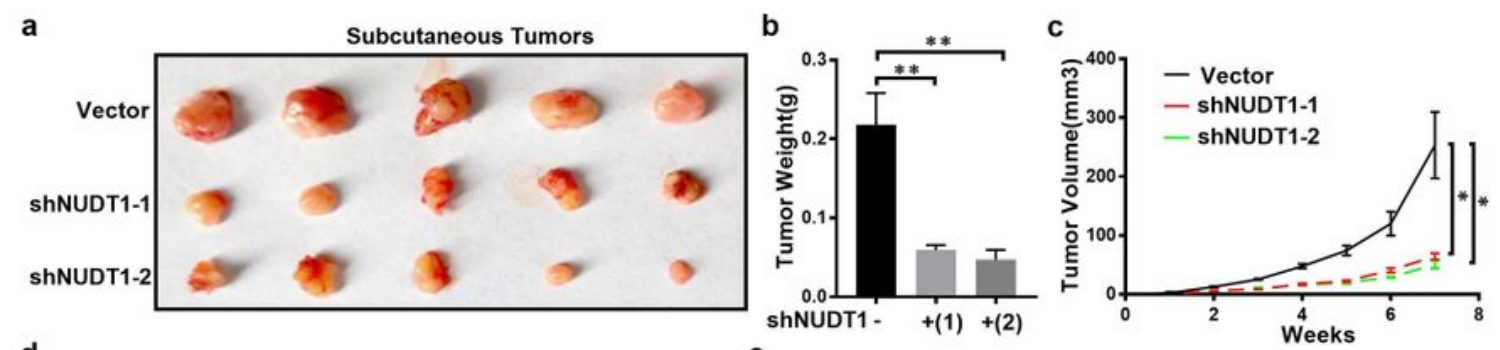

d

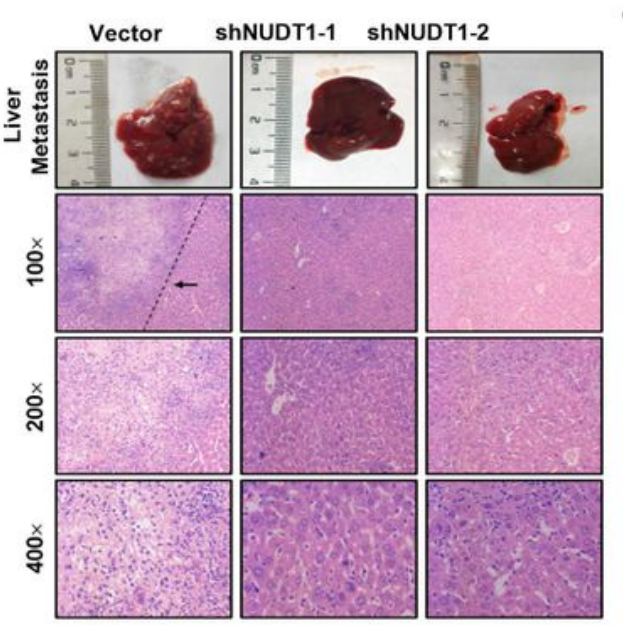

e

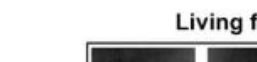

iving fluorescence images

f

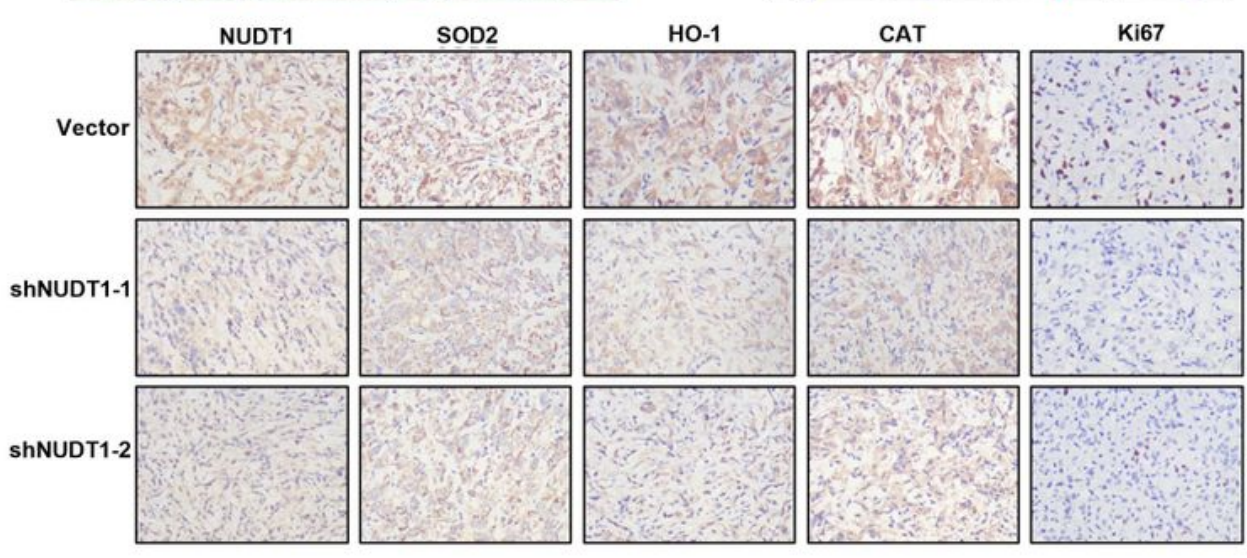

g

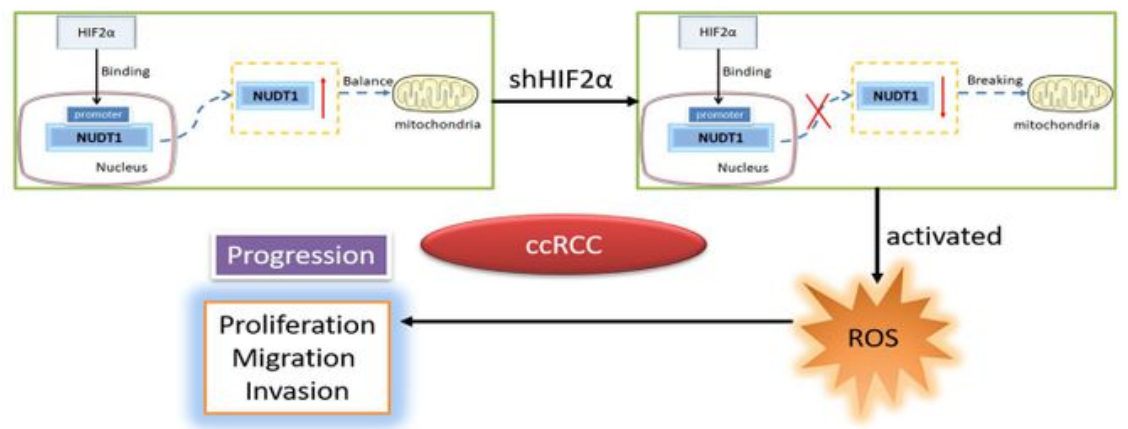

Figure 7 
Knockdown of NUDT1 suppresses ccRCC progression in vivo. (a-b) A498 cells and control cells transfected with shNUDT1-1 and shNUDT1-2 were injected subcutaneously into nude mice in the three groups. The tumor size and weight of mice in each group was measured after the seventh week. Data are expressed as the mean \pm SEM from tumors of each group. $* \star \star * ~ P<0.0001$, $* \star \star P<0.001$, $* * P<0.01$, and * $P<0.05$ (c) The tumor volume of each group was measured every week. This graph is drawn based on the relationship between the number of weeks after tumor cell implantation and tumor size $(\mathrm{mm} 3)$. Data are expressed as the mean \pm SEM from tumors of each group. ${ }^{\star \star \star \star} P<0.0001$, $* \star * P<0.001, * \star P<0.01$, and * $P<0.05$. (d) H\&E staining of liver tissue in the NUDT1 knockdown group and control group. (e) Living fluorescence images of the NUDT1 knockdown in the metastasis model group and control group. (f) Immunohistochemical (IHC) staining for NUDT1, markers of oxidative stress-related molecules (SOD2, HO-1, and CAT) and tumor malignancy (Ki67) in tumor xenografts. (g) A model in which HIF2a acts as a transcription factor to directly increase NUDT1 expression by binding to the HIF2a response element in the NUDT1 promoter. The high expression of HIF2a in ccRCC cells can directly target the promotion of NUDT1 expression to balance the mitochondrial metabolism in tumor cells and inhibit oxidative stress, thereby promoting the progress of ccRCC. When HIF2a is knocked down, it can target the reduction of NUDT1 expression, leading to increased ROS in tumor cells to cause the increase of oxidative stress level and inhibiting the occurrence and development of ccRCC.

\section{Supplementary Files}

This is a list of supplementary files associated with this preprint. Click to download.

- SUPPLEMENTALMATERIAL.docX

- SUPPLEMENTALMATERIAL.docx 